\title{
Poynting vector controversy in axion modified electrodynamics
}

\author{
Michael E. Tobar $\odot,{ }^{*}$ Ben T. McAllister, and Maxim Goryachev \\ ARC Centre of Excellence for Engineered Quantum Systems and ARC Centre of Excellence for Dark \\ Matter Particle Physics, Department of Physics, University of Western Australia, \\ 35 Stirling Highway, Crawley, Western Australia 6009, Australia
}

(Received 9 September 2021; accepted 28 January 2022; published 15 February 2022)

\begin{abstract}
The most sensitive haloscopes that search for axion dark matter through the two photon electromagnetic anomaly convert axions into photons through the mixing of axions with a large background direct current (DC) magnetic field. In this work we apply the Poynting theorem to the resulting axion modified electrodynamics and identify two possible Poynting vectors, one which is similar to the Abraham Poynting vector in electrodynamics and the other to the Minkowski Poynting vector. Inherently the conversion of axions to photons is a nonconservative process with respect to the created oscillating photonic degree of freedom. We show that the Minkowski Poynting theorem picks up the added nonconservative terms while the Abraham does not. The nonconservative terms may be categorized more generally as "curl forces," which in classical physics are nonconservative and nondissipative forces localized in space, not describable by a scalar potential and exist outside the global conservative physical equations of motion. To understand the source of energy conversion and power flow in the detection systems, we apply the two different Poynting theorems to both the resonant cavity haloscope and the broadband low-mass axion haloscope. Our calculations show that both Poynting theorems give the same sensitivity for a resonant cavity axion haloscope, but predict markedly different sensitivity for the low-mass broadband capacitive haloscope. Hence we ask the question, can understanding which one is the relevant one for axion dark matter detection be considered under the framework of the Abraham-Minkowski controversy? In reality, this should be confirmed by experiment when the axion is detected. However, many electrodynamic experiments have ruled in favor of the Minkowski Poynting vector when considering the canonical momentum in dielectric media. In light of this, we show that the axion modified Minkowski Poynting vector should indeed be taken seriously for sensitivity calculation for low-mass axion haloscopes in the quasistatic limit, and predict orders of magnitude better sensitivity than the Abraham Poynting vector equivalent.
\end{abstract}

DOI: 10.1103/PhysRevD.105.045009

\section{INTRODUCTION}

Axions are postulated to exist as neutral spin-zero bosons to solve the strong charge-parity problem in QCD. Such a particle is predicted to couple very weakly to other known particles and has thus been postulated to be cold dark matter [1-13]. In particular, many experiments rely on the electromagnetic anomaly, which is a two photon coupling term with the axion. To gain a significant sensitivity, it is widely considered that the best way to search for the axion is when the first background photonic degree of freedom is a large direct current (DC) magnetic field, which generates a second photon that can be detected. This is the basis of the

\footnotetext{
*michael.tobar@uwa.edu.au
}

Published by the American Physical Society under the terms of the Creative Commons Attribution 4.0 International license. Further distribution of this work must maintain attribution to the author(s) and the published article's title, journal citation, and DOI. Funded by SCOAP ${ }^{3}$.
DC magnetic field axion haloscope, first proposed by Sikivie $[14,15]$ and pioneered experimentally by the ADMX Collaboration [16-22]. Recently the scientific case that dark matter may include QCD axions or axionlike particles of varied mass and photon coupling has gained momentum [23-34], leading to many new ideas and new detector designs worldwide, which implement the principle of dark matter detection through the electromagnetic anomaly [35-76].

Inherently, the conversions of axions among a DC magnetic background field into a second photonic degree of freedom is a nonconservative process with respect to the second degree of freedom. In this process, the axion mixes with the background field to create a source term that drives the energy of conversion in the second photonic degree of freedom at a frequency corresponding to the axion mass. In this work, we implement the Poynting theorem to understand the source of energy conversion and flow in the system when considering axion modified electrodynamics.

In standard electrodynamics, Poynting vector analysis is implemented in circuit and antenna theory to understand 
how the input source power is impressed into the system along with the system power flow and how it relates to the stored energy and losses [77-81]. In this work we undertake a similar analysis within the framework of axion modified electrodynamics. In other work, the Poynting vector has been implemented with versions of the stress-energy tensor to understand energy and forces in magnetic and dielectric matter. For example, forces in systems, such as optical tweezers [82-85] and trapping of particles [86,87], where the best way to analyze these systems has been a subject of controversy (known as the Abraham-Minkowski controversy), is still an active area of debate [88-94]. This debate has led to the general concept of "curl forces," which are abundant in nature, cannot be described from the gradient of a scalar potential, and only exist in a localized space $[82,83,86,95]$. For example, the force on a particle with complex electric polarizability is known not to be derivable from a scalar potential as its curl is nonzero. Such forces are nonconservative and nondissipative, and their inclusion has been described both classically and quantum mechanically [96,97], in particular the quantizing of electrodynamics in dielectric and dispersive media [98-100]. Note such nonconservative curl forces do not include the most well-known curl force, which is the magnetic Lorentz force, as it is a conservative force that can do no work [96], described by a magnetic vector potential.

With this in mind, it has become evident that it is possible to derive alternative versions of Poynting's theorem (in fact, four versions are possible) [101]. In particular, the Minkowski Poynting vector [102], $\vec{S}_{D B}=\frac{1}{\epsilon_{0} \mu_{0}} \vec{D} \times \vec{B}$, has been shown to be successful to account for experiments in dielectric media, where the field momentum is associated with the canonical momentum $[89,92,94,103]$; here the electric flux density, $\vec{D}=\epsilon_{0} \vec{E}+\vec{P}$, is the sum of the electric field, $\vec{E}$, and electric polarization, $\vec{P}$, and the magnetic flux density, $\vec{B}=\mu_{0}(\vec{H}+\vec{M})$, is the sum of the magnetic field, $\vec{H}$, and magnetization, $\vec{M}$. Naturally, when the curl of the polarization is nonzero $(\nabla \times \vec{P} \neq 0)$ the Minkowski Poynting vector will pick up this term, due to an unconventional but necessary modification to Faraday's law [98,101], while the Abraham Poynting vector [104,105], $\vec{S}_{E H}=\vec{E} \times \vec{H}$, will not.

For the curl of the polarization to be nonzero, an energy input is required to separate the bound charge; this describes a permanent electret or energy harvesting material [106] as well as the properties of ferroelectric domain walls [107]. This description is also similar to an active dipole in antenna theory, a voltage source in circuit theory, or an active dipole emitter in quantum theory [98-100], where an external nonconservative force (sometimes referred as a fictitious or pseudo force) is described by an impressed electric field (sometimes referred as a fictitious or pseudo electric field [108-110]) with a nonzero curl (one could call this a polarization). Furthermore, the electret, energy harvester, or ferroelectric domain may be classified as an active bound charge dipole. We may recognize this active dipole term generally as a nonconservative curl force term, which necessarily modifies Faraday's law, and is only present internally to the active antenna, voltage source, electret, or ferroelectric domain and not present globally outside the active device. As with all curl forces, this nonconservative term cannot be characterized by a scalar potential; on the other hand, it has recently been shown to be characterized via an electric vector potential $[62,98,99,106,111,112]$.

Recently, it was also shown that there exists a similar nonconservative curl force term in axion modified electrodynamics [62,112]. This occurs when the axion mixes with a DC background magnetic field, which converts the axion mass to the energy of the second photonic degree of freedom [62,112]. In this representation the axion mixing with the DC magnetic field adds a similar term to a polarization with a nonzero curl $[106,111]$. In this work we apply the Minkowski and Abraham Poynting theorem equivalents to axion modified electrodynamics and compare the difference, where the former picks up the extra curl force term, while the latter does not.

\section{THE EFFECTIVE AXION CURRENT AND CHARGE DENSITY}

It is well known that axions modify electrodynamics through the axion two photon coupling [31,113], which in vacuum leads to the following set of modified Maxwell's equations:

$\nabla \cdot \vec{E}=\frac{\rho_{e}}{\varepsilon_{0}}+c g_{a \gamma \gamma} \vec{B} \cdot \nabla a$,

$\nabla \times \vec{B}-\frac{1}{c^{2}} \partial_{t} \vec{E}=\mu_{0} \vec{J}_{e}-g_{a \gamma \gamma} \mu_{0} \epsilon_{0} c\left(\vec{B} \partial_{t} a+\nabla a \times \vec{E}\right)$,

$\nabla \cdot \vec{B}=0$

$\nabla \times \vec{E}+\partial_{t} \vec{B}=0$.

Here $g_{\text {ary }}$ is the two-photon coupling to an axion field, $a(t)$ is the amplitude of the axion field, $\rho_{e}$ is the volume charge density, and $\vec{J}_{e}$ is the volume current density. One common way to set up the equations of motion for the two photon interaction is to assume $\nabla a=0$, so two of the three terms go to zero and only one modification to Ampere's law remains,

$$
\nabla \times \vec{B}-\frac{1}{c^{2}} \partial_{t} \vec{E}=\mu_{0}\left(\vec{J}_{e}-g_{a \gamma \gamma} \epsilon_{0} c \vec{B} \partial_{t} a\right),
$$

where the axion current is defined by

$$
\vec{J}_{a}=-g_{a \gamma \gamma} \epsilon_{0} c \vec{B} \partial_{t} a .
$$

This modification is commonly used in the calculation of the sensitivity of haloscope experiments. 
A more general version of the modifications as source terms can be obtained by substituting the following vector identities: $\vec{B} \cdot \nabla a=\nabla \cdot(a \vec{B})-a(\nabla \cdot \vec{B})$ and $\nabla a \times \vec{E}=$ $\nabla \times(a \vec{E})-a(\nabla \times \vec{E})$ into (1). Then, assuming to first order $\nabla \cdot \vec{B}=0$ and $\nabla \times \vec{E}=-\partial_{t} \vec{B}$, the modified Gauss' and Ampere's laws may be written as [31,112]

$$
\begin{aligned}
\epsilon_{0} \nabla \cdot \vec{E} & =\rho_{e}+\rho_{a b}, \\
\frac{1}{\mu_{0}} \nabla \times \vec{B}-\epsilon_{0} \partial_{t} \vec{E} & =\vec{J}_{e}+\vec{J}_{a b}+\vec{J}_{a e},
\end{aligned}
$$

where

$$
\begin{aligned}
& \rho_{a b}=g_{a \gamma \gamma} \epsilon_{0} c \nabla \cdot(a(t) \vec{B}(\vec{r}, t)), \\
& \vec{J}_{a b}=-g_{a \gamma \gamma} \epsilon_{0} c \partial_{t}(a(t) \vec{B}(\vec{r}, t)), \\
& \vec{J}_{a e}=-g_{a \gamma \gamma} \epsilon_{0} c \nabla \times(a(t) \vec{E}(\vec{r}, t)) .
\end{aligned}
$$

Here, $\vec{J}_{a b}$ is similar to a polarization current, $\rho_{a b}$ is similar to a bound charge, and they are related through the continuity equation

$$
\nabla \cdot \vec{J}_{a b}=-\partial_{t} \rho_{a b}
$$

Furthermore, $\vec{J}_{a e}$ is similar to a bound current, so the total axion current is thus $\vec{J}_{a}=\vec{J}_{a b}+\vec{J}_{a e}$, which is a more general form of Eq. (3). Note that setting these terms to zero because $\nabla a=0$ at the beginning of a calculation that analyzes the sensitivity of an axion-photon coupled system can result in missing some parts of the solution [62,112], as we show in the next section.

\section{AXION MODIFIED ELECTRODYNAMICS}

\section{A. Time dependent form}

Rather that write the equation of motion with modified source terms, as in (4) and (5), we may include the modifications in the definitions of the fields themselves in a similar way to the auxiliary fields in matter [112]. With some rearrangement of these equations, we can show $[31,106,114]$

$$
\begin{aligned}
& \nabla \cdot\left(\vec{E}(\vec{r}, t)-g_{a \gamma \gamma} a(t) c \vec{B}(\vec{r}, t)\right)=\frac{\rho_{e}}{\epsilon_{0}},
\end{aligned}
$$

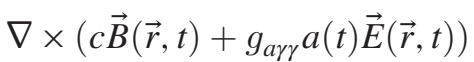

$$
\begin{aligned}
& -\frac{1}{c} \partial_{t}\left(\vec{E}(\vec{r}, t)-g_{\text {ary }} a(\vec{r}, t) c \vec{B}(\vec{r}, t)\right)=c \mu_{0} \vec{J}_{e}, \\
& \nabla \cdot c \vec{B}(\vec{r}, t)=0, \\
& \nabla \times \vec{E}(\vec{r}, t)+\frac{1}{c} \partial_{t} c \vec{B}(\vec{r}, t)=0 .
\end{aligned}
$$

This has been shown to be equivalent to a perturbative transformation of the electromagnetic fields $[112,115,116]$, given by

$$
\begin{aligned}
c \vec{B}^{\prime}(\vec{r}, t) & \rightarrow c \vec{B}(\vec{r}, t)+g_{a \gamma \gamma} a(t) \vec{E}(\vec{r}, t) \quad \text { and } \\
\vec{E}^{\prime}(\vec{r}, t) & \rightarrow \vec{E}(\vec{r}, t)-g_{a \gamma \gamma} a(t) c \vec{B}(\vec{r}, t),
\end{aligned}
$$

where Eqs. (8) and (9) in the quasistatic limit effectively represent dual symmetry with respect to a rotation angle,

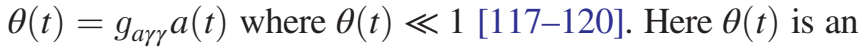
effective dynamical pseudoscalar field, which in this case is the product of the axion pseudoscalar field, $a(t)$, with the axion photon coupling, $g_{a \gamma \gamma}$. For dark matter axions, $a(t)$ is in general a large classical field; however, $\theta(t)$ remains small due to the extremely weak coupling of axions to photons, i.e., $g_{a \gamma \gamma} \ll 1$. Note that there is also a duality transformation between electromagnetic potentials, where the dual 4-vector potential contains a magnetic scalar potential and an electric vector potential. Under this duality transform the electric vector potential manifests [116-120], which potentially adds the axion induced curl force to the system under investigation. This is evident from Eq. (9), as the curl of $\vec{E}_{1}^{\prime}$ has a nonzero spatial term.

Now considering the interaction includes two photons, we distinguish between a background field (denoted by subscript 0 ) and the generated photon field (denoted by subscript 1), which is created by the axion pseudoscalar field mixing with the background field. To first order we may assume the background field satisfies Maxwell's equations, so that

$$
\begin{aligned}
& \nabla \times \vec{B}_{0}=\mu_{0} \epsilon_{0} \partial_{t} \vec{E}_{0}+\mu_{0} \vec{J}_{e_{0}}, \\
& \nabla \times \vec{E}_{0}=-\partial_{t} \vec{B}_{0}, \\
& \nabla \cdot \vec{B}_{0}=0 \\
& \nabla \cdot \vec{E}_{0}=\epsilon_{0}^{-1} \rho_{e_{0}} .
\end{aligned}
$$

Note that any axion modification of the background field will end up second order with respect to the effects on the second generated photonic degree of freedom, so it can be ignored [31,114].

Thus for the generated photonic degree of freedom, we may write (7) in a similar way to how the auxiliary fields are included in Maxwell's equations in matter, and we find axion modified electrodynamics in a familiar form [112], given by

$$
\begin{aligned}
& \nabla \cdot \vec{D}_{1}=\rho_{e 1}, \\
& \nabla \times \vec{H}_{1}-\partial_{t} \vec{D}_{1}=\vec{J}_{e 1}, \\
& \nabla \cdot \vec{B}_{1}(\vec{r}, t)=0, \\
& \nabla \times \vec{E}_{1}(\vec{r}, t)+\partial_{t} \vec{B}_{1}(\vec{r}, t)=0,
\end{aligned}
$$


where (8) and (9) are akin to the following constitutive relations:

$$
\begin{aligned}
\vec{H}_{1}(\vec{r}, t) & =\frac{\vec{B}_{1}}{\mu_{0}}-\vec{M}_{1}-\vec{M}_{1 a} \text { and } \\
\vec{D}_{1}(\vec{r}, t) & =\epsilon_{0} \vec{E}_{1}+\vec{P}_{1}+\vec{P}_{1 a} .
\end{aligned}
$$

Here, $\vec{M}_{1}$ and $\vec{P}_{1}$ are the nonaxion induced magnetization and polarization, respectively, while the axion modifications, $\vec{M}_{1 a}$ and $\vec{P}_{1 a}$, are moved to redefinitions of the auxiliary fields rather than source terms and to first order with respect to the background field are given by

$$
\begin{aligned}
\vec{M}_{1 a} & =-g_{a \gamma \gamma} a(t) c \epsilon_{0} \vec{E}_{0}(\vec{r}, t) \quad \text { and } \\
\frac{1}{\epsilon_{0}} \vec{P}_{1 a} & =-g_{a \gamma \gamma} a(t) c \vec{B}_{0}(\vec{r}, t) .
\end{aligned}
$$

Here it is clear the divergence of $\vec{M}_{1 a}$ is nonzero, similar to what occurs at the boundaries of a permanent magnet, the curl of $\vec{P}_{1 a}$ is nonzero similar to what occurs at the boundaries of a permanent electret, and by combining (13) and (10) it can be calculated to be (assuming $\nabla a=0$ )

$$
\begin{aligned}
\nabla \cdot \vec{M}_{1 a} & =-g_{a \gamma \gamma} a(t) c \epsilon_{0} \nabla \cdot \vec{E}_{0}(\vec{r}, t)=-g_{a \gamma \gamma} a(t) c \rho_{e_{0}}, \\
\frac{1}{\epsilon_{0}} \nabla \times \vec{P}_{1 a} & =-g_{a \gamma \gamma} a(t) c \nabla \times \vec{B}_{0}(\vec{r}, t) \\
& =-\frac{g_{a \gamma \gamma} a(t)}{c} \partial_{t} \vec{E}_{0}-g_{a \gamma \gamma} a(t) c \mu_{0} \vec{J}_{e_{0}} .
\end{aligned}
$$

Note that if we followed the procedure to set $\nabla a=0$ at the start of the calculation, then the axion current in (3) would be the only modification, and the general form of the modified constitutive relations in (12) would be missed. This would be akin to falsely setting $\nabla \times \vec{P}_{1 a}=0$ and $\nabla$. $\vec{M}_{1 a}=0$ even though they are in general nonzero in the approximation when $\nabla a$ is set to zero.

Assuming only a DC background magnetic field, $\vec{B}_{0}(\vec{r})$ with no background electric field $\left(\vec{E}_{0}=0\right.$ and $\left.\vec{M}_{1 a}=0\right)$ as well as in vacuum $\left(\vec{M}_{1}=0, \vec{P}_{1}=0, \vec{B}_{1}=\mu_{0} \vec{H}_{1}\right)$, one can write the axion modified Ampere's law from Eq. (11) as

$$
\nabla \times \vec{B}_{1}=\mu_{0} \partial_{t} \vec{D}_{1}+\mu_{0} \vec{J}_{e_{1}} .
$$

In contrast, Faraday's law with respect to the $\vec{E}_{1}$ and $\vec{B}_{1}$ fields remains unchanged,

$$
\nabla \times \vec{E}_{1}=-\partial_{t} \vec{B}_{1} .
$$

However, given the fact for the DC case the curl of $\vec{P}_{1 a}$ in Eq. (14) is nonzero $\left[\frac{1}{\epsilon_{0}} \nabla \times \vec{P}_{1 a}=-g_{\text {ary }} a(t) c \mu_{0} \vec{J}_{e_{0}}\right.$, a modified Faraday's law may be written in a similar fashion as to what is undertaken with an electret or impressed voltage source when the curl is nonzero $[98,99,101,106]$, so by taking the curl of $\vec{D}_{1}$ in (12) and combing with (16) we obtain

$$
\frac{1}{\epsilon_{0}} \nabla \times \vec{D}_{1}=-\partial_{t} \vec{B}_{1}-g_{a \gamma \gamma} a \mu_{0} c \vec{J}_{e_{0}},
$$

which is analogous to an electromagnetic system in matter where the curl of the polarization is nonzero. It has been shown in such systems the fundamental electromagnetic quantities become the electric $\vec{D}$ and magnetic $\vec{B}$ flux densities [98,101,106], which is compatible with the Minkowski Poynting vector.

In this work we apply these more general equations to low-mass axion haloscopes, which necessarily include the impressed current, $\vec{J}_{e_{0}}$, which creates the background magnetic field $\vec{B}_{0}(\vec{r})$. Note that there also exists a dual symmetry with the source terms in the above Eqs. (15) and (17), where an effective impressed magnetic current manifests through the axion interaction with the impressed electrical current, $\vec{J}_{e_{0}}$, so $\vec{J}_{m_{1}}^{\prime}(\vec{r}, t) \rightarrow g_{a \gamma \gamma} a(t) \mu_{0} c \vec{J}_{e_{0}}(\vec{r}, t)$. The fact that this impressed magnetic current exists does not necessitate the existence of free magnetic monopoles, in the same way bound currents and polarization currents do not need the existence of free electrons or any other free charge carrier. For example, bound magnetic monopoles exist in nature as permanent magnets consisting of bound north and south pole pairs, which can be set in motion, with a net bound magnetic current if one pole is kept stationary as the other rotates. Such a rotating magnet converts the mechanical motion to an electromotive force with nonzero curl (a curl force) [106]. This fact has been recognised as early as 1936 [121], where Schelkunoff from Bell Labs stated, "It is true that there are no magnetic conductors and no magnetic conduction currents in the same sense as there are electric conductors and electric conduction currents but magnetic convection currents are just as real as electric convection currents, although the former exist only in doublets of oppositely directed currents since magnetic charges themselves are observable only in doublets."

\section{B. Harmonic phasor form}

For harmonic solutions of the axion-Maxwell equations we write the equations in complex vector-phasor form. For example, we set $\vec{E}_{1}(\vec{r}, t)=\frac{1}{2}\left(\mathbf{E}_{1}(\vec{r}) e^{-j \omega_{1} t}+\right.$ $\left.\mathbf{E}_{1}^{*}(\vec{r}) e^{j \omega_{1} t}\right)=\operatorname{Re}\left[\mathbf{E}_{1}(\vec{r}) e^{-j \omega_{1} t}\right]$, so we define the vector phasor (bold) and its complex conjugate by $\tilde{\mathbf{E}}_{1}(\vec{r}, t)=$ $\mathbf{E}_{1}(\vec{r}) e^{-j \omega_{1} t}$ and $\tilde{\mathbf{E}}_{1}^{*}(\vec{r}, t)=\mathbf{E}_{1}^{*}(\vec{r}) e^{j \omega_{1} t}$, respectively. In contrast, the axion pseudoscalar field, $a(t)$, may be written as $a(t)=\frac{1}{2}\left(\tilde{a} e^{-j \omega_{a} t}+\tilde{a}^{*} e^{j \omega_{a} t}\right)=\operatorname{Re}\left(\tilde{a} e^{-j \omega_{a} t}\right)$, and thus, in phasor form, $\tilde{A}=\tilde{a} e^{-j \omega_{a} t}$ and $\tilde{A}^{*}=\tilde{a}^{*} e^{j \omega_{a} t}$. Thus, the phasor form of the modified Ampere's law in (15) becomes 


$$
\begin{aligned}
\frac{1}{\mu_{0}} \nabla \times \tilde{\mathbf{B}}_{1} & =\tilde{\mathbf{J}}_{e_{1}}-j \omega_{1} \epsilon_{0} \tilde{\mathbf{E}}_{1}+j \omega_{a} g_{a \gamma \gamma} \epsilon_{0} c \tilde{A} \vec{B}_{0}, \\
\frac{1}{\mu_{0}} \nabla \times \tilde{\mathbf{B}}_{1}^{*} & =\tilde{\mathbf{J}}_{e_{1}}^{*}+j \omega_{1} \epsilon_{0} \tilde{\mathbf{E}}_{1}^{*}-j \omega_{a} g_{a \gamma \gamma} \epsilon_{0} c \tilde{A}^{*} \vec{B}_{0},
\end{aligned}
$$

while the phasor form of Faraday's law in (16) becomes

$$
\begin{aligned}
& \nabla \times \tilde{\mathbf{E}}_{1}=j \omega_{1} \tilde{\mathbf{B}}_{1}, \\
& \nabla \times \tilde{\mathbf{E}}_{1}^{*}=-j \omega_{1} \tilde{\mathbf{B}}_{1}^{*},
\end{aligned}
$$

and the phasor form of the modified Faraday's law in (17) becomes

$$
\begin{aligned}
& \frac{1}{\epsilon_{0}} \nabla \times \tilde{\mathbf{D}}_{1}=j \omega_{1} \tilde{\mathbf{B}}_{1}-g_{a \gamma \gamma} c \mu_{0} \tilde{A} \vec{J}_{e_{0}}, \\
& \frac{1}{\epsilon_{0}} \nabla \times \tilde{\mathbf{D}}_{1}^{*}=-j \omega_{1} \tilde{\mathbf{B}}_{1}^{*}-g_{a \gamma \gamma} c \mu_{0} \tilde{A}^{*} \vec{J}_{e_{0}}^{*} .
\end{aligned}
$$

In the following we use these equations to calculate energy and power via the Poynting theorem in a DC magnetic field axion haloscope.

\section{CALCULATION OF POWER GENERATED IN A DC MAGNETIC FIELD AXION HALOSCOPE USING POYNTING THEOREM}

We start by considering the instantaneous Poynting vector in its standard physics textbook form of

$$
\begin{aligned}
\vec{S}_{1}(t) & =\frac{1}{\mu_{0}} \vec{E}_{1}(t) \times \vec{B}_{1}(t) \\
& =\frac{1}{2}\left(\mathbf{E}_{1} e^{-j \omega_{1} t}+\mathbf{E}_{1}^{*} e^{j \omega_{1} t}\right) \times \frac{1}{2 \mu_{0}}\left(\mathbf{B}_{1} e^{-j \omega_{1} t}+\mathbf{B}_{1}^{*} e^{j \omega_{1} t}\right) \\
& =\frac{1}{2 \mu_{0}} \operatorname{Re}\left(\mathbf{E}_{1} \times \mathbf{B}_{1}^{*}\right)+\frac{1}{2 \mu_{0}} \operatorname{Re}\left(\mathbf{E}_{1} \times \mathbf{B}_{1} e^{-j 2 \omega_{1} t}\right), \quad(21)
\end{aligned}
$$

which consists of a DC term, the first term on the right-hand side of (21), and a high frequency term, the second term on the right-hand side of (21). Note that the DC term in (21) is equivalent to the time average of the instantaneous Poynting vector.

Thus, the complex Poynting vector and its complex conjugate are defined by

$$
\mathbf{S}_{1}=\frac{1}{2 \mu_{0}} \mathbf{E}_{1} \times \mathbf{B}_{1}^{*} \quad \text { and } \quad \mathbf{S}_{1}^{*}=\frac{1}{2 \mu_{0}} \mathbf{E}_{1}^{*} \times \mathbf{B}_{1},
$$

respectively, where $\mathbf{S}_{1}$ is the complex power density of the harmonic electromagnetic wave or oscillation, with the real part equal to the time averaged power density and the imaginary term equal to the reactive power, which may be inductive (magnetic energy dominates) or capacitive (electrical energy dominates).
In the case that the complex Poynting vector is only real, then the $\mathbf{E}_{1}$ and $\mathbf{B}_{1}$ fields are in phase, which describes a propagating wave with distinct direction and momentum. Such a propagating wave can be generated by an antenna in the far-field limit, at distances larger than the wavelength of the emitted photon, and is a source of loss from the antenna. Another case where $\mathbf{E}_{1}$ and $\mathbf{B}_{1}$ are in phase is due to resistive losses: in this case the photon energy is converted to heat and destroyed; however, both are effectively loss terms with respect to the antenna, the former known as radiation loss. Conversely, in the near field limit of an antenna, the Poynting vector is imaginary as $\mathbf{E}_{1}$ and $\mathbf{B}_{1}$ are out of phase. This represents reactive energy flow between the antenna power source and the antenna near field, which exists at subwavelength distances from the antenna. In this case the photons do not propagate away from the antenna, and they exist as quasistatic oscillating $\mathbf{E}_{1}$ and $\mathbf{B}_{1}$ fields with no net momentum.

A convenient and unambiguous way to calculate the real and imaginary parts of the Poynting vector is through the following equations:

$\operatorname{Re}\left(\mathbf{S}_{1}\right)=\frac{1}{2}\left(\mathbf{S}_{1}+\mathbf{S}_{1}^{*}\right)$ and $j \operatorname{Im}\left(\mathbf{S}_{1}\right)=\frac{1}{2}\left(\mathbf{S}_{1}-\mathbf{S}_{1}^{*}\right)$.

We use these equations in the following to calculate the real and imaginary parts of the complex Poynting vector in axion modified electrodynamics.

\section{A. Axion modified Minkowski Poynting theorem}

Based on the axion modified $\vec{D}_{1}$ vector, we may calculate the complex axion modified Minkowski Poynting vector in a similar way to Eq. (22), which is given by

$\mathbf{S}_{D B}=\frac{1}{2 \epsilon_{0} \mu_{0}} \mathbf{D}_{1} \times \mathbf{B}_{1}^{*} \quad$ and $\quad \mathbf{S}_{D B}^{*}=\frac{1}{2 \epsilon_{0} \mu_{0}} \mathbf{D}_{1}^{*} \times \mathbf{B}_{1}$.

Taking the divergence of Eq. (24) we find

$$
\begin{aligned}
\nabla \cdot \mathbf{S}_{D B} & =\frac{1}{2} \nabla \cdot\left(\frac{1}{\epsilon_{0}} \mathbf{D}_{1} \times \frac{1}{\mu_{0}} \mathbf{B}_{1}^{*}\right) \\
& =\frac{1}{2}\left(\frac{1}{\mu_{0}} \mathbf{B}_{1}^{*} \cdot \frac{1}{\epsilon_{0}} \nabla \times \mathbf{D}_{1}-\frac{1}{\epsilon_{0}} \mathbf{D}_{1} \cdot \frac{1}{\mu_{0}} \nabla \times \mathbf{B}_{1}^{*}\right)
\end{aligned}
$$

and

$$
\begin{aligned}
\nabla \cdot \mathbf{S}_{D B}^{*} & =\frac{1}{2} \nabla \cdot\left(\frac{1}{\epsilon_{0}} \mathbf{D}_{1}^{*} \times \frac{1}{\mu_{0}} \mathbf{B}_{1}\right) \\
& =\frac{1}{2}\left(\frac{1}{\mu_{0}} \mathbf{B}_{1} \cdot \frac{1}{\epsilon_{0}} \nabla \times \mathbf{D}_{1}^{*}-\frac{1}{\epsilon_{0}} \mathbf{D}_{1}^{*} \cdot \frac{1}{\mu_{0}} \nabla \times \mathbf{B}_{1}\right) .
\end{aligned}
$$

Combining (25) and (26) with (20), (18), and (23) along with the divergence theorem (this is a standard technique in microwave engineering and circuit theory 
$[79,101,122,123]$ ), after some calculation we obtain (see Appendix A for details)

$$
\begin{aligned}
& \oint \operatorname{Re}\left(\mathbf{S}_{D B}\right) \cdot \hat{n} d s \\
& =\int\left(\frac{j\left(\omega_{1}-\omega_{a}\right)}{4} \epsilon_{0} g_{a \gamma \gamma} c \vec{B}_{0} \cdot\left(\tilde{a} \mathbf{E}_{1}^{*}-\tilde{a}^{*} \mathbf{E}_{1}\right)\right. \\
& \quad+\frac{1}{4} g_{a \gamma \gamma} c \vec{B}_{0} \cdot\left(\tilde{a} \mathbf{J}_{e_{1}}^{*}+\tilde{a}^{*} \mathbf{J}_{e_{1}}\right)-\frac{1}{4} g_{a \gamma \gamma} \vec{J}_{e_{0}} \cdot\left(\tilde{a}^{*} c \mathbf{B}_{1}+\tilde{a} c \mathbf{B}_{1}^{*}\right) \\
& \left.\quad-\frac{1}{4}\left(\mathbf{E}_{1} \cdot \mathbf{J}_{e_{1}}^{*}+\mathbf{E}_{1}^{*} \cdot \mathbf{J}_{e_{1}}\right)\right) d V
\end{aligned}
$$

and

$$
\begin{aligned}
\oint & j \operatorname{Im}\left(\mathbf{S}_{D B}\right) \cdot \hat{n} d s \\
= & \int\left(\frac{j \omega_{1}}{2}\left(\frac{1}{\mu_{0}} \mathbf{B}_{1}^{*} \cdot \mathbf{B}_{1}-\epsilon_{0} \mathbf{E}_{1} \cdot \mathbf{E}_{1}^{*}\right)\right. \\
& +\frac{j\left(\omega_{1}+\omega_{a}\right) \epsilon_{0} g_{a \gamma \gamma}}{4} c \vec{B}_{0} \cdot\left(\tilde{a} \mathbf{E}_{1}^{*}+\tilde{a}^{*} \mathbf{E}_{1}\right) \\
& +\frac{1}{4} g_{a \gamma \gamma} c \vec{B}_{0} \cdot\left(\tilde{a} \mathbf{J}_{e_{1}}^{*}-\tilde{a}^{*} \mathbf{J}_{e_{1}}\right)+\frac{1}{4} g_{a \gamma \gamma} \vec{J}_{e_{0}} \cdot\left(\tilde{a}^{*} c \mathbf{B}_{1}-\tilde{a} c \mathbf{B}_{1}^{*}\right) \\
& \left.-\frac{1}{4}\left(\mathbf{E}_{1} \cdot \mathbf{J}_{e_{1}}^{*}-\mathbf{E}_{1}^{*} \cdot \mathbf{J}_{e_{1}}\right)\right) d V .
\end{aligned}
$$

The closed surface integral on the left-hand side of (27) is the time averaged power radiated from inside to outside the haloscope volume, which for a closed system such as a cavity will be zero. However, for an open system such as a radio frequency antenna, real power will radiate in the far field. In contrast, the closed surface integral on the left-hand side of (28) is the reactive power radiated outside the haloscope volume, which in general does not have to be zero, in a similar way to how reactive power oscillates to and from the voltage source charging and discharging a reactive capacitor in circuit theory, or the reactive power in an antenna, where energy oscillates from the antenna to the near field and then is reabsorbed by the antenna, due to the antenna's self-capacitance or inductance.

\section{B. Axion modified Abraham Poynting theorem}

The complex Abraham pointing vector is basically the same as Eq. (22) for the case we are considering with $\vec{H}_{1}=\frac{1}{\mu_{0}} \vec{B}_{1}$. Taking the divergence of Eq. (22) we find

$$
\begin{aligned}
\nabla \cdot \mathbf{S}_{\mathrm{EH}} & =\frac{1}{2} \nabla \cdot\left(\mathbf{E}_{1} \times \mathbf{H}_{1}^{*}\right) \\
& =\frac{1}{2} \mathbf{H}_{1}^{*} \cdot\left(\nabla \times \mathbf{E}_{1}\right)-\frac{1}{2} \mathbf{E}_{1} \cdot\left(\nabla \times \mathbf{H}_{1}^{*}\right)
\end{aligned}
$$

and

$$
\begin{aligned}
\nabla \cdot \mathbf{S}_{\mathrm{EH}}^{*} & =\frac{1}{2} \nabla \cdot\left(\mathbf{E}_{1}^{*} \times \mathbf{H}_{1}\right) \\
& =\frac{1}{2} \mathbf{H}_{1} \cdot\left(\nabla \times \mathbf{E}_{1}^{*}\right)-\frac{1}{2} \mathbf{E}_{1}^{*} \cdot\left(\nabla \times \mathbf{H}_{1}\right) .
\end{aligned}
$$

Combining (29) and (30) with (19), (18), and (23) along with the divergence theorem, we obtain (see Appendix A for details)

$$
\begin{aligned}
\oint \operatorname{Re}\left(\mathbf{S}_{E H}\right) \cdot \hat{n} d s= & \int\left(\frac{j \omega_{a}}{4} \epsilon_{0} g_{a \gamma \gamma} c \vec{B}_{0} \cdot\left(\tilde{a}^{*} \mathbf{E}_{1}-\tilde{a} \mathbf{E}_{1}^{*}\right)\right) \\
& \left.-\frac{1}{4}\left(\mathbf{E}_{1} \cdot \mathbf{J}_{e_{1}}^{*}+\mathbf{E}_{1}^{*} \cdot \mathbf{J}_{e_{1}}\right)\right) d V
\end{aligned}
$$

and

$$
\begin{aligned}
\oint j \operatorname{Im}\left(\mathbf{S}_{E H}\right) \cdot \hat{n} d s= & \int\left(\frac{j \omega_{1}}{2}\left(\mu_{0} \mathbf{H}_{1}^{*} \cdot \mathbf{H}_{1}-\epsilon_{0} \mathbf{E}_{1} \cdot \mathbf{E}_{1}^{*}\right)\right. \\
& +\frac{j \omega_{a}}{4} \epsilon_{0} g_{a \gamma \gamma} c \vec{B}_{0} \cdot\left(\tilde{a}^{*} \mathbf{E}_{1}+\tilde{a} \mathbf{E}_{1}^{*}\right) \\
& \left.\left.-\frac{1}{4}\left(\mathbf{E}_{1} \cdot \mathbf{J}_{e_{1}}^{*}-\mathbf{E}_{1}^{*} \cdot \mathbf{J}_{e_{1}}\right)\right)\right) d V
\end{aligned}
$$

As before, the closed surface integral on the left-hand side of (31) and (32) is the time averaged power and reactive power radiated outside the haloscope volume, respectively. However, the Abraham Poynting vector misses three extra terms the Minkowski Poynting vector picks up, due to the inclusion of the nonconservative and nondissipative source term described by Eq. (20).

\section{Abraham or Minkowski Poynting theorem in axion modified electrodynamics?}

Currently most calculations of haloscope detection sensitivity a priori assume the Abraham Poynting vector is valid, with the exception of one or two $[62,112]$. However, as shown in the Minkowski-Abraham debate over the past century, this is not "clear-cut" and in a stationary dielectric media where the canonical momentum is under consideration, the Minkowski form agrees with experimental results $[89,92,94]$. This may be true in axion modified electrodynamics, as we can identify a similar guilty term due to Eq. (14) and hence (17). The nonzero curl should do active work without adding dissipation and should not be ignored in calculations of axion detector sensitivity. This extra term has all the properties of a curl force $[82,83,95]$, which adds spatial terms to the Poynting vector equations. In the following sections we compare the two ways of determining the power of photonic conversion for various axion haloscope topologies.

\section{RESONANT CAVITY HALOSCOPE}

In this section we derive the sensitivity of an ADMX style radio frequency haloscope based on a cavity resonator [14-22], with a schematic shown in Fig. 1. First, we 
undertake the calculation using the Abraham Poynting vector, as this is the a priori Poynting vector assumed across most of the literature, and then we compare and contrast calculations using the Minkowski Poynting vector.

For a power source, $P_{s}$, delivering energy to a resonator as shown in Fig. 1, the resonance is defined when the reactive power delivered by the source is zero, and thus when tuned on resonance the circulating energy only oscillates between the electric and the magnetic energy in the resonator at the cavity resonance frequency, with no energy oscillating between the cavity and the power source (which in this case is the axion mixing with a DC magnetic field). In this case the power delivered to the cavity is real. This corresponds to the real part of the Poynting theorem equations, which we use in the next section to calculate the sensitivity of the axion haloscope. Internal to the cavity resonator, this circulating energy is described by a reactive (or imaginary) Poynting vector, which causes the power in the resonator to build up, with respect to the source input power, $P_{s}$. This buildup is limited by the dissipation in the resonator and hence $Q$-factor. The buildup of circulating power is given by $P_{c}=Q_{1} P_{s}$, where in the steady state $P_{s}=P_{d}$, which is also related to the stored energy in the resonator, $U_{1}$, by $P_{s}=\omega_{1} U_{1}$.

Thus, in such a cavity resonator the electric and magnetic field are out of phase (as opposed to a propagating wave, which is in phase), and in this paper we represent the lossless electric field vector phasor as real and the lossless magnetic field vector phasor as imaginary (and so the cross product is imaginary). Dissipative terms, whether calculated in the volume or on the surface assume Ohm's law, dictate that the dissipative part of the electric field be in phase with surface or volume currents and hence the magnetic field. Thus, the electric field effectively gains an imaginary component when losses are included. However, the majority of the electric field is real, with $\operatorname{Im}\left(\vec{E}_{1}\right) \sim-\operatorname{Re}\left(\vec{E}_{1}\right) / Q_{1}$. The

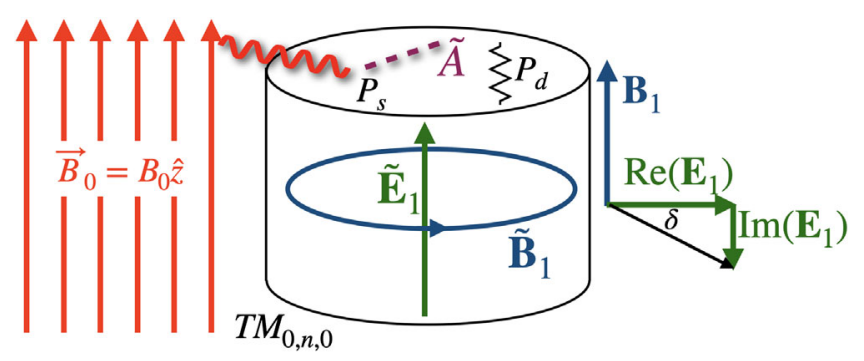

FIG. 1. Schematic of axion conversion in an ADMX style haloscope. Such haloscopes are based on radio frequency cavity resonators, where an external DC magnetic field, $\vec{B}_{0}$, has a nonzero dot product with the nondissipative electric field of the radio frequency mode in the cavity, $\operatorname{Re}\left(\mathbf{E}_{1}\right)$. For a cylindrical cavity the sensitive modes are from the $T M_{0, n, 0}$ mode family, where $n$ is the radial mode number. The axial and azimuthal mode numbers must be zero. Finite losses mean part of the electric field, $\operatorname{Im}\left(\mathbf{E}_{1}\right)$, is in phase with the magnetic field, $\mathbf{B}_{1}$, characterized by the loss angle $\delta$, which for a high- $Q$ system is very small and related by $\tan \delta \sim \frac{1}{Q_{1}}$. tangential real part of the electric field must be continuous at the cavity wall boundary, which for a perfect conductor is zero and sets the boundary conditions to calculate the frequency of the electromagnetic modes. Setting the reactive power to zero on resonance, allows us to calculate if there is any frequency shift of the bare cavity when excited by axions. We do find a small second order in the $Q$-factor effect, calculated using Foster's reactance theorem [124]. However, there is no major impact on the sensitivity calculation. For completeness this is detailed in Appendix B and predicts a different value of frequency shift depending on whether we use the Minkowski or the Abraham Poynting theorem.

\section{A. Cavity dissipated power}

Both Poynting theorems have a dissipative term in the real components, listed as the final term on the right-hand side of Eqs. (27) and (31) and graphically shown in Fig. 1. For dissipation effects over the volume, the volume current is in phase with the imaginary part of the electric field and is of the form $\mathbf{J}_{e_{1}}=\sigma_{e} \mathbf{E}_{1}$, where $\sigma_{e}$ is the effective conductivity of the volume, which is related to the loss tangent of the volume by $\sigma_{e}=\omega_{a} \epsilon_{0} \tan \delta$ and given $\frac{1}{Q_{1}} \sim \tan \delta$, then $\mathbf{J}_{e_{1}} \sim \frac{\omega_{a} \epsilon_{0}}{Q_{1}} \mathbf{E}_{1}$, substituting these values in the last term on the right-hand side of Eq. (27) or (31), the dissipated power in the cavity is calculated to be

$$
P_{d}=\frac{\omega_{a} \epsilon_{0}}{2 Q_{1}} \int \mathbf{E}_{1} \cdot \mathbf{E}_{1}^{*} d V=\frac{\omega_{a} U_{1}}{Q_{1}} .
$$

For surface loss, the same volume integrals given by (27) and (31) collapse to surface integrals, where the surface current on the cavity walls is represented by the vector phasor, $\mathbf{K}_{1}=\hat{n} \times \mathbf{H}_{1}$, of dimensions amps/meter and the electric field at the surface is nonzero and in phase with the surface current, related by $\mathbf{E}_{1}=R_{S} \mathbf{K}_{1}$, where $R_{S}$ is the surface resistance. Again, substituting these values in the last term on the right-hand side of Eq. (27) or (31) means the dissipated power in the cavity is

$P_{d}=\frac{R_{S}}{2} \oint \mathbf{K}_{1} \cdot \mathbf{K}_{1}^{*} d s=\frac{R_{S}}{2} \oint \mathbf{H}_{1} \cdot \mathbf{H}_{1}^{*} d s=\frac{\omega_{a} U_{1}}{Q_{1}}$,

which gives the same relationship with respect to the stored energy, $U_{1}$, and the dissipated power, whether it dissipates over the volume or over the surface of the cavity resonator.

\section{B. Sensitivity from the Abraham Poynting theorem}

In this calculation we assume the cavity is a closed system so there is no real power radiating outside the cavity volume, and this means the closed surface integral on the left-hand side of (31) should be set to zero $\left[\oint \operatorname{Re}\left(\mathbf{S}_{E H}\right) \cdot \hat{n} d s=0\right]$. In practice power is taken outside the cavity due to the coupling, which in effect loads the cavity $Q$-factor, and this phenomenon may be added after the calculation using standard techniques. We also assume that the axion and the resonator 
frequency coincide $\left(\omega_{1}=\omega_{a}\right)$, and therefore the magnetic and electric energy inside the resonator will be equal, where again the effects of detuning may be added using standard techniques. Under these assumptions Eq. (31) becomes

$P_{s}=\frac{j g_{a \gamma \gamma} \omega_{a} c}{4} \int \vec{B}_{0} \cdot\left(\tilde{a}^{*} \epsilon_{0} \mathbf{E}_{1}-\tilde{a} \epsilon_{0} \mathbf{E}_{1}^{*}\right) d V=P_{d}=\frac{\omega_{a} U_{1}}{Q_{1}}$.

Here, $P_{s}$ is the axion source power and must be real, note the source power is equal to the dissipated power, and as calculated in the last section it can occur over the volume and/or over the cavity surface.

For the source power to be nonzero either $\mathbf{E}_{1}$ or the axion scalar field, $\tilde{a}$, has to be imaginary. Since the axion scalar field is assumed to be lossless, we consider only the former to be imaginary, as has been suggested previously [114]. The general complex electric field is of the form $\mathbf{E}_{1} \approx(1-j \tan \delta) \operatorname{Re}\left(\mathbf{E}_{1}\right)$ in the regime where the loss angle is very small, $\delta \ll 1$. Hence, the axion source term in the steady state becomes

$$
P_{s}=\frac{g_{a \gamma \gamma} a_{0} \omega_{a} \epsilon_{0} c}{2 Q_{1}} \int \vec{B}_{0} \cdot \operatorname{Re}\left(\mathbf{E}_{1}(\vec{r})\right) d V
$$

where $a_{0}=\frac{1}{2}\left(\tilde{a}+\tilde{a}^{*}\right)$ is the peak value of the scalar axion field, so $a_{0}=\sqrt{2}\left\langle a_{0}\right\rangle$. Equating (36) to $P_{d}=\frac{\omega_{a} U_{1}}{Q_{1}}$ derived in (33) or (34) gives

$U_{1}=\frac{g_{a \gamma \gamma} a_{0} \epsilon_{0} c}{2} \int \vec{B}_{0} \cdot \operatorname{Re}\left(\mathbf{E}_{1}\right) d V=\frac{\epsilon_{0}}{2} \int \mathbf{E}_{1} \cdot \mathbf{E}_{1}^{*} d V$.

Now defining the form factor of the cavity haloscope as

$$
C_{1}=\frac{\left(\int \vec{B}_{0} \cdot \operatorname{Re}\left(\mathbf{E}_{1}\right) d V\right)^{2}}{B_{0}^{2} V_{1} \int \mathbf{E}_{1} \cdot \mathbf{E}_{1}^{*} d V},
$$

the axion induced circulating power may be calculated to be

$$
\begin{aligned}
P_{1} & =\omega_{a} Q U_{1}=g_{a \gamma \gamma}^{2}\left\langle a_{0}\right\rangle^{2} \omega_{a} Q_{1} \epsilon_{0} c^{2} B_{0}^{2} V_{1} C_{1} \\
& =g_{a \gamma \gamma}^{2} \rho_{a} Q_{1} \epsilon_{0} c^{5} B_{0}^{2} V_{1} C_{1} \frac{1}{\omega_{a}},
\end{aligned}
$$

where $\left\langle a_{0}\right\rangle^{2}=\frac{\rho_{a}}{c} \frac{\hbar^{2}}{m_{a}^{2}}$ and $\rho_{a}$ is the axion dark matter density. This calculation is consistent with what has been derived previously $[15,31,36]$.

\section{Sensitivity from the Minkowski Poynting theorem}

As before, assuming the real power inside the cavity haloscope is a closed system $\left[\oint \operatorname{Re}\left(\mathbf{S}_{D B}\right) \cdot \hat{n} d s=0\right]$, the cavity is embedded inside a magnet $\left[\vec{J}_{e_{0}} \cdot\left(\tilde{a} c \mathbf{B}_{1}^{*}+\right.\right.$ $\left.\tilde{a}^{*} c \mathbf{B}_{1}\right)=0$ ], and the axion and the resonator frequency coincide $\left(\omega_{1}=\omega_{a}\right)$. Then, in this case Eq. (27) becomes

$P_{s}=\int \frac{1}{4} g_{a \gamma \gamma} c \vec{B}_{0} \cdot\left(\tilde{a} \mathbf{J}_{e_{1}}^{*}+\tilde{a}^{*} \mathbf{J}_{e_{1}}\right) d V=P_{d}=\frac{\omega_{a} U_{1}}{Q_{1}}$.

Here, $P_{s}$ is the axion source power and must be real.

As undertaken in the Abraham technique, we assume a lossy volume current in phase with the electric field of the form $\mathbf{J}_{e_{1}}=\sigma_{e} \mathbf{E}_{1}$ where $\sigma_{e}=\frac{\omega_{a} \epsilon_{0}}{Q_{1}}$. Substituting the same value of $\mathbf{J}_{e_{1}}$ into (40) gives

$$
P_{s}=\frac{g_{a \gamma \gamma} a_{0} \omega_{a} \epsilon_{0} c}{2 Q_{1}} \int \vec{B}_{0} \cdot \operatorname{Re}\left(\mathbf{E}_{1}(\vec{r})\right) d V
$$

the same as calculated for the Abraham technique in Eq. (36), which means both Eqs. (38) and (39) are calculable using both the Minkowski and the Abraham Poynting vectors, and are consistent with previous sensitivity calculations for a standard ADMX style haloscope.

\section{LOW-MASS BROADBAND AXION HALOSCOPES UNDER DC MAGNETIC FIELD}

For a low-mass broadband detector in the quasistatic limit, a haloscope may be inductive or capacitive and must be driven by reactive power from the source, so in the first approximation any dissipation or radiation loss can be ignored and is thus set to zero. As before, we consider the generated electric field to be real $\left(\mathbf{E}_{1}^{*}=\mathbf{E}_{1}\right)$ and the out of phase magnetic field as imaginary $\left(\mathbf{B}_{1}^{*}=-\mathbf{B}_{1}\right)$. Also, conduction currents will be in the same phase as the magnetic field and hence imaginary $\left(\mathbf{J}_{e_{1}}^{*}=-\mathbf{J}_{e_{1}}\right)$. In this case, it is clear that the real part of the delivered complex Poynting vector given by (31) and (27) must be zero, and the sensitivity of the reactive low-mass broadband haloscope will be determined from the imaginary reactive power delivered by the axion interacting with the background DC magnetic field.

There has been some recent controversy in the calculation of sensitivity for low mass reactive experiments in the quasistatic limit, where the majority of the publications suggest that the sensitivity to electric field is suppressed when the Compton wavelength of the axion is larger than the experimental dimensions [40,114,125,126]. These experiments assume that the only modification to Maxwell's equations is due to the axion current (3), which is equivalent to assuming no boundary or spatial effects and thus setting the total derivative to zero. On the other hand, it has been shown that making these approximations too early in the calculation can lead to valid solutions being lost $[62,112,127]$ due to extra spatial or surface terms, which in this case is due to the fact that $\vec{P}_{1 a}=-g_{a \gamma \gamma} a(t) \epsilon_{0} c \vec{B}_{0}(\vec{r})$ has a nonzero curl, which can also be thought as a connection to the Witten effect [128]. Based on this, more sensitive experiments have been proposed using inductive wire loop readouts [62], or 
capacitive parallel plate readouts $[112,129]$. In the following, as an example, we compare the sensitivity of a parallel plate capacitor to low mass axions by implementing both Poynting vector theorems.

\section{A. Capacitor under DC magnetic field}

For a parallel plate capacitor as shown in Fig. 2 the last terms on the right-hand side of Eqs. (32) and (28) must be zero, since the conduction current must be zero in the lossless capacitor volume. This also means the third last term on the right-hand side of (28) must be zero. Furthermore, if we assume the capacitor is embedded inside a DC magnet, the second last term in (28) must also be zero (it is possible to make use of this term to make a sensitive low-mass detector [62]). This means the equations for reactive power flowing into and out of the capacitor volume, using the Abraham and Minkowski forms, are given by

$$
\begin{aligned}
\oint j \operatorname{Im}\left(\mathbf{S}_{E H}\right) \cdot \hat{n} d s= & j \omega_{a} \int\left(\frac{1}{2 \mu_{0}} \mathbf{B}_{1}^{*} \cdot \mathbf{B}_{1}-\frac{\epsilon_{0}}{2} \mathbf{E}_{1}^{*} \cdot \mathbf{E}_{1}\right. \\
& \left.+\frac{\epsilon_{0}}{2} g_{a \gamma \gamma} a_{0} c \vec{B}_{0} \cdot \operatorname{Re}\left(\mathbf{E}_{1}\right)\right) d V
\end{aligned}
$$

and

$$
\begin{aligned}
\oint j \operatorname{Im}\left(\mathbf{S}_{D B}\right) \cdot \hat{n} d s= & j \omega_{a} \int\left(\frac{1}{2 \mu_{0}} \mathbf{B}_{1}^{*} \cdot \mathbf{B}_{1}-\frac{\epsilon_{0}}{2} \mathbf{E}_{1}^{*} \cdot \mathbf{E}_{1}\right. \\
& \left.+\epsilon_{0} g_{a \gamma \gamma} a_{0} c \vec{B}_{0} \cdot \operatorname{Re}\left(\mathbf{E}_{1}\right)\right) d V
\end{aligned}
$$

respectively.

For the capacitor in Fig. 2 the alternating current (AC) electric field phasor, ignoring fringing, is of the form

$$
\mathbf{E}_{1}=\frac{\tilde{q}_{1}}{\pi R_{c}^{2} \epsilon_{0}} \hat{z}
$$

where $\tilde{q}_{1}$ is the complex phasor of electric charge on the capacitor plates. Following this, from Ampere's law the AC

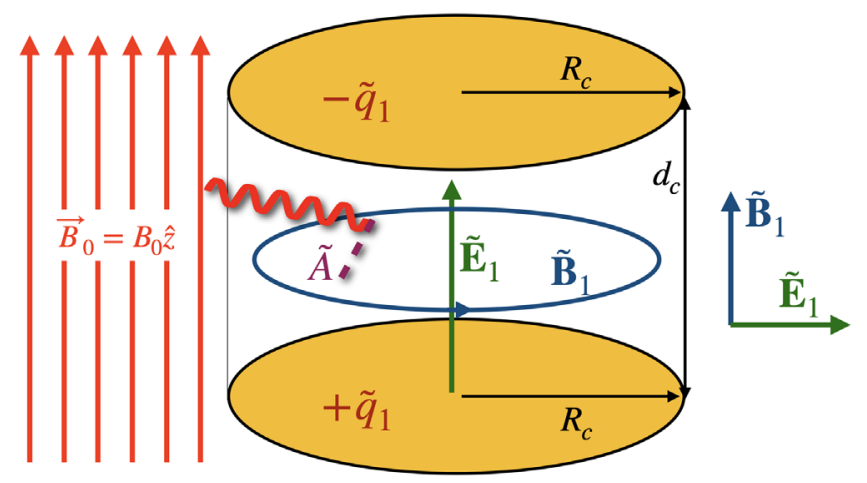

FIG. 2. Schematic of axion conversion in a capacitive haloscope. magnetic field phasor within the capacitor volume $\left(V_{c}=\pi R_{c}^{2} d_{c}\right)$ may be calculated to be

$$
\mathbf{B}_{1}=-j \omega_{a} \mu_{0} \tilde{q}_{1} \frac{r}{\pi R_{c}^{2}} \hat{\theta} .
$$

Following this we may calculate the ratio of the magnetic energy density to electric energy density in the capacitor given by

$$
\frac{\mathbf{B}_{1} \cdot \mathbf{B}_{1}^{*}}{\epsilon_{0} \mu_{0} \mathbf{E}_{1} \cdot \mathbf{E}_{1}^{*}}=\frac{r^{2} \omega_{a}^{2}}{4 c^{2}}=\frac{\pi^{2} r^{2}}{\lambda_{a}^{2}}
$$

where $\lambda_{a}$ is the Compton wavelength of the axion. Integrating over the volume of the capacitor allows us to calculate the ratio of magnetic to electric energy to be (ignoring fringing)

$$
\frac{\int_{V_{c}} \mathbf{B}_{1} \cdot \mathbf{B}_{1}^{*} d V}{\epsilon_{0} \mu_{0} \int_{V_{c}} \mathbf{E}_{1} \cdot \mathbf{E}_{1}^{*} d V}=\frac{R_{c}^{2} \omega_{a}^{2}}{8 c^{2}}=\frac{\pi^{2} R_{c}^{2}}{2 \lambda_{a}^{2}} .
$$

These equations highlight that at DC the parallel plate capacitor is purely capacitive, but at $\mathrm{AC}$ the capacitor has a small but finite inductance in the quasistatic limit, when $\lambda_{a}>R_{c}$. When $\lambda_{a} \sim R_{c}$, the capacitor could become resonant, similar to a $T M_{0,1,0}$ mode in a cylindrical cavity; however, this would not be in the quasistatic limit. Nevertheless, in a circuit where the direction of the electric field $\mathbf{E}_{1}$ in a capacitor is parallel to the applied DC magnetic field, $\overrightarrow{B_{0}}$, Eq. (37) still holds for the capacitor, with an effective form factor of unity, which can be shown by substituting Eq. (44) into (38), and we can use this fact to help calculate the sensitivity of a low-mass capacitor experiment.

\section{Sensitivity assuming the Abraham Poynting theorem}

Assuming the Abraham Poynting theorem, the reactive power delivered to and from a capacitor under the DC magnetic field as shown in Fig. 2 can be calculated by substituting Eq. (37) into (42), and using (46) we find

$$
\begin{aligned}
j P_{a} & =\oint j \operatorname{Im}\left(\mathbf{S}_{E H}\right) \cdot \hat{n} d s \\
& =\frac{j \omega_{a} g_{a \gamma \gamma} a_{0} \epsilon_{0} c}{2} \int\left(\vec{B}_{0} \cdot \operatorname{Re}\left(\mathbf{E}_{1}\right)\right) \frac{\pi^{2} r^{2}}{\lambda_{a}^{2}} d V .
\end{aligned}
$$

Now, from the definition of the haloscope form factor (38), the reactive power delivered to the capacitor given by (48) becomes

$P_{a}=\omega_{a} U_{c}, \quad$ where $U_{c}=g_{a \gamma \gamma}^{2}\left\langle a_{0}\right\rangle^{2} \epsilon_{0} c^{2} B_{0}^{2} V_{1}\left(\frac{\pi^{2} R c^{2}}{2 \lambda_{a}^{2}}\right)^{2}$. 
Thus the magnitude of the voltage phasor across the capacitor can be calculated from $U_{c}=\frac{1}{2} \tilde{\mathcal{V}} \tilde{\mathcal{V}}^{*} C_{a}$ $\left(C_{a}=\frac{\pi R_{c}^{2} \epsilon_{0}}{d_{c}}\right)$ to be

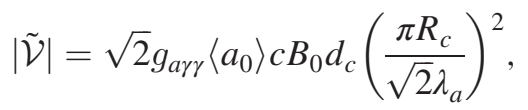

which is consistent with an rms voltage across the capacitor of

$$
\begin{aligned}
\mathcal{V}_{\mathrm{rms}} & =g_{a \gamma \gamma}\left\langle a_{0}\right\rangle c B_{0} d_{c}\left(\frac{\pi R_{c}}{\sqrt{2} \lambda_{a}}\right)^{2} \\
& =g_{a \gamma \gamma} d_{c} \frac{c}{\omega_{a}} B_{0} \sqrt{\rho_{a} c^{3}}\left(\frac{\pi R_{c}}{\sqrt{2} \lambda_{a}}\right)^{2},
\end{aligned}
$$

where $\left\langle a_{0}\right\rangle=\sqrt{\frac{\rho_{a}}{c}} \frac{\hbar}{m_{a}}$ and $\rho_{a}$ is the axion dark matter density. This calculation is consistent with other calculations based on just the axion current $[114,125,126]$, as given by Eq. (3); However, it does not take into account the nonzero value of the curl of $\vec{P}_{1 a}$. The calculation predicts suppressed sensitivity at low mass, proportional to $\frac{R_{c}^{2}}{\lambda_{a}^{2}}$.

\section{Sensitivity assuming the Minkowski Poynting theorem}

Assuming the Minkowski Poynting theorem, the reactive power delivered to and from a capacitor under the DC magnetic field as shown in Fig. 2 can be calculated by substituting Eq. (37) into (43). Note that in this case the magnetic energy is insignificant so ignoring this component gives

$$
\begin{aligned}
j P_{a} & =\oint j \operatorname{Im}\left(\mathbf{S}_{D B}\right) \cdot \hat{n} d s \\
& =\frac{j \omega_{a} g_{a \gamma \gamma} a_{0} \epsilon_{0} c}{2} \int\left(\vec{B}_{0} \cdot \operatorname{Re}\left(\mathbf{E}_{1}\right)\right) d V .
\end{aligned}
$$

Now, from the definition of the haloscope energy (37) and form factor (38), the energy stored in the capacitor (52) becomes

$$
U_{c}=g_{a \gamma \gamma}^{2}\left\langle a_{0}\right\rangle^{2} \epsilon_{0} c^{2} B_{0}^{2} V_{1} .
$$

Thus the magnitude of the voltage phasor across the capacitor can be calculated from $U_{c}=\frac{1}{2} \tilde{\mathcal{V}} \tilde{\mathcal{V}}^{*} C_{a}$ to be

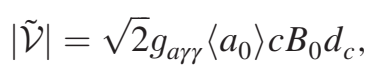

which is consistent with an rms voltage of

$$
\mathcal{V}_{\mathrm{rms}}=g_{a \gamma \gamma}\left\langle a_{0}\right\rangle c B_{0} d_{c}=g_{a \gamma \gamma} d_{c} \frac{c}{\omega_{a}} B_{0} \sqrt{\rho_{a} c^{3}},
$$

which is the same as calculated previously [129]. Thus, we may conclude, from the Minkowski Poynting theorem, a sensitive low-mass experiment may be undertaken using a capacitive haloscope.

\section{DISCUSSION AND CONCLUSIONS}

By applying the Poynting theorem to axion modified electrodynamics, we have shown how the sensitivity of a resonant cavity and reactive broadband axion haloscope may be calculated. However, the way we apply the theorem is dependent on the type of detector. For example, the Poynting vector analysis had already been undertaken to calculate the sensitivity of the MADMAX detector [43-46]. However, MADMAX is in the regime where the Compton wavelength of the axion is much smaller than the detector size, and it is thus in a different regime from the resonant and reactive haloscope discussed in this paper. The MADMAX detector converts energy at a dielectric boundary and is assumed to be in the propagating wave (or far field) limit, where the $\tilde{\mathbf{E}}$ and $\tilde{\mathbf{B}}$ vector phasors are in phase, so the Poynting vector is real, represents the physical energy flux leaving a surface, propagates through the haloscope [43-45], and in principle can be made broadband.

In contrast, the resonant haloscope is generally the size of the Compton wavelength of the axion (unless higher order modes are implemented) and thus has an imaginary Poynting vector internally within the resonator. This is because the axion induced photon energy produced within the resonator is reflected at the resonator boundaries, so the energy is localized in the form of a standing wave, with the $\tilde{\mathbf{E}}$ and $\tilde{\mathbf{B}}$ fields out of phase. In this work we have assumed the electric field is real, and thus the magnetic field is imaginary. However, on resonance (when $\omega_{a}=\omega_{1}$ ), the axion conversion process within the resonant cavity haloscope does not need to supply any reactive power, only real power. In this case the real part of the Poynting vector equation has both a source term and a dissipative term within the cavity, which are equal in the steady state, allowing the incident source power to escape the volume as heat, through the resistive losses. Meanwhile, reactive power flow oscillates between the electric and the magnetic fields within the cavity. The higher the $Q$-factor the more the circulating power builds up within the cavity, meaning the percentage of dissipation per cycle is smaller, and hence the detector sensitivity is proportional to the $Q$-factor. The down side is that the technique is narrow band, which requires complicated tuning mechanisms to scan for the axion of unknown mass.

On the other hand, low-mass broadband experiments are in the quasistatic regime, where the Compton wavelength is much greater than the dimensions of the detector. In this case the sensitivity is determined by the reactive power flow within the detector created from the axion-photon conversion. For the higher frequency resonant cavity haloscope, we have shown that the implementation of either the Minkowski or the Abraham axion modified Poynting vector has no significant influence on the calculated sensitivity. In contrast, for lowmass reactive haloscopes there is a large difference in sensitivity calculated from the two Poynting theorems.

Currently, the Minkowski-Abraham controversy in electrodynamics interacting with matter is considered to be resolved 
by identifying the Abraham and Minkowski Poynting vector with the total system kinetic and canonical momentum, respectively [94,130]. For electrodynamics in matter, the Abraham Poynting theorem is the correct one to use when the whole dielectric body is displaced together as a solid entity [89]. In contrast, the Minkowski Poynting vector is the relevant one to use when considering the canonical momentum, which acts to spatially translate particles within the dielectric [103], such as bound charge, which may in some cases cause the curl of the polarization to be nonzero [98101,106,107]. For example, the conservation law for the canonical momentum has been validated through atomic recoil in spontaneous emission [103].

For axion modified electrodynamics the Minkowski solution suggests a similar nonconservative effect under a DC background magnetic field, $\vec{B}_{0}(\vec{r})$, because from Eq. (14), when $\nabla a=0$, the curl of $\vec{P}_{1 a}$ is nonzero and is given by $\nabla \times \vec{P}_{1 a}=-g_{a \gamma \gamma} a(t) c \epsilon_{0} \nabla \times \vec{B}_{0}(\vec{r})=-g_{a \gamma \gamma} a(t) \epsilon_{0} \mu_{0} c \vec{J}_{e_{0}}$. This term is created at the surface defined by the coil winding where the impressed electric current, $\vec{J}_{e_{0}}$, flows, which is the source term that creates $\vec{B}_{0}(\vec{r})$. The curl of $\vec{P}_{1 a}$ may also be described as a fictitious oscillating magnetic current boun-

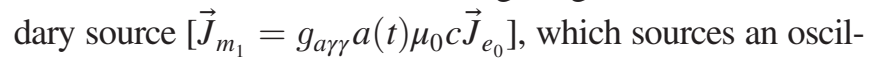
lating spatial nonconservative electric curl force (or emf), with a force per unit charge of $-g_{a \gamma \gamma} a(t) c \vec{B}_{0}$, which displaces the axion charge harmonically in time, creating a polarization current of $\vec{J}_{a}=\partial_{t} \vec{P}_{1 a}=-g_{a \gamma \gamma} \epsilon_{0} c \vec{B}_{0} \partial_{t} a$, which is equivalent to the axion current given by Eq. (3). Here, the harmonic spatial translation of axion charge creates the oscillating photonic degree of freedom through the QCD axion-photon anomaly in an analogous way to photons created via spontaneous emission from dipole emitters in [103]. The nonconservative boundary source gives an unsuppressed sensitivity at low mass proportional to $g_{a \gamma \gamma} a(t)$, whereas experiments proportional to the axion current are suppressed through the time derivative.

Recently, this effect has also been shown to be apparent in topological insulators, where a material with a polarization of nonzero curl was shown to be associated with a magnetic current boundary (or instanton), a Berry phase and nonzero crystal momentum [131]. Moreover, they showed a nonzero static $\theta$ angle angle was possible because a nonzero magnetoelectric angle in 3D, does not obstruct the gauge invariance of polarization density, and $\theta$ can be interpreted as the magnetoelectric polarizability, i.e., a magnetic field induces an extra polarization density, $\Delta \vec{P}$ equivalent to Eq. (13) with a static value of $\theta[132,133]$.

For axion modified electrodynamics the Abraham solution is consistent with the total derivative equal to zero, which is the prevailing view among the axion dark matter community. For the total derivative to be zero, it is well known that all surface terms must go to zero as the Compton wavelength approaches infinity (surfaces are essentially assumed to go to infinity). In this case the axion current is the only source term, which contributes to observable effects. In this work we have challenged this view through the Abraham-Minkowski controversy, while putting forward the idea that the conversion from the axion scalar field to power in the oscillating photonic degree of freedom is just another way to generate photonic power (or electricity) from an external nonphotonic degree of freedom. In this case the underlying microscopic mechanism is the axion-photon anomaly from QCD, where the surrounding ensemble of axions from dark matter mix with the virtual photons from the DC magnetic field to supply the external energy that will generate power in the oscillating photonic degree of freedom.

In the end, to verify which description gives the correct solution will require experimentation, which will only be possible when the axion is discovered.

\section{ACKNOWLEDGMENTS}

This work was funded by the ARC Centre of Excellence for Engineered Quantum Systems, CE170100009, and the ARC Centre of Excellence for Dark Matter Particle Physics, CE200100008. B. M. was also funded by the Forrest Research Foundation.

\section{APPENDIX A: DERIVATION OF POYNTING THEOREM EQUATIONS}

In this Appendix we derive Eqs. (27), (28), (31), and (32) in the main text.

\section{Axion modified Minkowski Poynting theorem}

To derive Eq. (27) and (28), we begin with writing the divergence of the real and imaginary parts of $\mathbf{S}_{D B}$ as

$$
\begin{aligned}
\nabla \cdot \operatorname{Re}\left(\mathbf{S}_{D B}\right) & =\frac{1}{2} \nabla \cdot\left(\mathbf{S}_{D B}+\mathbf{S}_{D B}^{*}\right), \\
\nabla \cdot \operatorname{Im}\left(\mathbf{S}_{D B}\right) & =\frac{1}{2} \nabla \cdot\left(\mathbf{S}_{D B}-\mathbf{S}_{D B}^{*}\right) .
\end{aligned}
$$

The next step is to calculate $\nabla \cdot \mathbf{S}_{D B}$ and $\nabla \cdot \mathbf{S}_{D B}^{*}$,

$$
\begin{aligned}
\nabla \cdot \mathbf{S}_{D B} & =\frac{1}{2} \nabla \cdot\left(\frac{1}{\epsilon_{0}} \mathbf{D}_{1} \times \frac{1}{\mu_{0}} \mathbf{B}_{1}^{*}\right) \\
& =\frac{1}{2}\left(\frac{1}{\mu_{0}} \mathbf{B}_{1}^{*} \cdot \frac{1}{\epsilon_{0}} \nabla \times \mathbf{D}_{1}-\frac{1}{\epsilon_{0}} \mathbf{D}_{1} \cdot \frac{1}{\mu_{0}} \nabla \times \mathbf{B}_{1}^{*}\right)
\end{aligned}
$$

and

$$
\begin{aligned}
\nabla \cdot \mathbf{S}_{D B}^{*} & =\frac{1}{2} \nabla \cdot\left(\frac{1}{\epsilon_{0}} \mathbf{D}_{1}^{*} \times \frac{1}{\mu_{0}} \mathbf{B}_{1}\right) \\
& =\frac{1}{2}\left(\frac{1}{\mu_{0}} \mathbf{B}_{1} \cdot \frac{1}{\epsilon_{0}} \nabla \times \mathbf{D}_{1}^{*}-\frac{1}{\epsilon_{0}} \mathbf{D}_{1}^{*} \cdot \frac{1}{\mu_{0}} \nabla \times \mathbf{B}_{1}\right) .
\end{aligned}
$$


In harmonic form, the axion modified Ampere's and Faraday's laws under a background DC $B$-field of $\vec{B}_{0}(\vec{r})$, created by an impressed electrical DC current in the magnet coil, $\vec{J}_{e_{0}}$, may be written as

$$
\begin{aligned}
& \frac{1}{\mu_{0}} \nabla \times \mathbf{B}_{1}=\mathbf{J}_{e_{1}}-j \omega_{1} \epsilon_{0} \mathbf{E}_{1}+j \omega_{a} g_{a \gamma \gamma} \epsilon_{0} c \vec{B}_{0} \tilde{a}, \\
& \frac{1}{\mu_{0}} \nabla \times \mathbf{B}_{1}^{*}=\mathbf{J}_{e_{1}}^{*}+j \omega_{1} \epsilon_{0} \mathbf{E}_{1}^{*}-j \omega_{a} g_{a \gamma \gamma} \epsilon_{0} c \vec{B}_{0} \tilde{a}^{*}, \\
& \frac{1}{\epsilon_{0}} \nabla \times \mathbf{D}_{1}=j \omega_{1} \mathbf{B}_{1}-g_{a \gamma \gamma} c \mu_{0} \tilde{a} \vec{J}_{e_{0}}, \\
& \frac{1}{\epsilon_{0}} \nabla \times \mathbf{D}_{1}^{*}=-j \omega_{1} \mathbf{B}_{1}^{*}-g_{a \gamma \gamma} c \mu_{0} \tilde{a}^{*} \vec{J}_{e_{0}} .
\end{aligned}
$$

Substituting Eq. (A4) into Eqs. (A2) and (A3) leads to

$$
\begin{aligned}
\nabla \cdot \mathbf{S}_{D B}= & \frac{1}{2 \mu_{0}} \mathbf{B}_{1}^{*} \cdot\left(j \omega_{1} \mathbf{B}_{1}-g_{a \gamma \gamma} \tilde{a} c \mu_{0} \vec{J}_{e_{0}}\right) \\
& -\frac{1}{2}\left(\mathbf{E}_{1}-g_{a \gamma \gamma} \tilde{a} c \vec{B}_{0}\right) \cdot\left(\mathbf{J}_{e_{1}}^{*}+j \omega_{1} \epsilon_{0} \mathbf{E}_{1}^{*}-j \omega_{a} g_{a \gamma \gamma} \epsilon_{0} \tilde{a}^{*} c \vec{B}_{0}\right) \\
= & \frac{j \omega_{1}}{2}\left(\frac{1}{\mu_{0}} \mathbf{B}_{1}^{*} \cdot \mathbf{B}_{1}-\epsilon_{0} \mathbf{E}_{1} \cdot \mathbf{E}_{1}^{*}\right)+\frac{j \omega_{1}}{2} \epsilon_{0} g_{a \gamma \gamma} \tilde{a} c \vec{B}_{0} \cdot \mathbf{E}_{1}^{*} \\
& +\frac{j \omega_{a}}{2} g_{a \gamma \gamma} \epsilon_{0} \tilde{a}^{*} c \vec{B}_{0} \cdot \mathbf{E}_{1}-\frac{1}{2} \mathbf{E}_{1} \cdot \mathbf{J}_{e_{1}}^{*}+\frac{1}{2} g_{a \gamma \gamma} \tilde{a} c \vec{B}_{0} \cdot \mathbf{J}_{e_{1}}^{*} \\
& -\frac{1}{2} g_{a \gamma \gamma} \tilde{a} c \mathbf{B}_{1}^{*} \cdot \vec{J}_{e_{0}}
\end{aligned}
$$

and

$$
\begin{aligned}
\nabla \cdot \mathbf{S}_{D B}^{*}= & \frac{1}{2 \mu_{0}} \mathbf{B}_{1} \cdot\left(-j \omega_{1} \mathbf{B}_{1}^{*}-g_{a \gamma \gamma} \tilde{a}^{*} c \mu_{0} \vec{J}_{e_{0}}\right) \\
& -\frac{1}{2}\left(\mathbf{E}_{1}^{*}-g_{a \gamma \gamma} \tilde{a}^{*} c \vec{B}_{0}\right) \cdot\left(\mathbf{J}_{e_{1}}-j \omega_{1} \epsilon_{0} \mathbf{E}_{1}+j \omega_{a} g_{a \gamma \gamma} \epsilon_{0} \tilde{a} c \vec{B}_{0}\right) \\
= & \frac{j \omega_{1}}{2}\left(\epsilon_{0} \mathbf{E}_{1} \cdot \mathbf{E}_{1}^{*}-\frac{1}{\mu_{0}} \mathbf{B}_{1}^{*} \cdot \mathbf{B}_{1}\right)-\frac{j \omega_{1}}{2} \epsilon_{0} g_{a \gamma \gamma} \tilde{a}^{*} c \vec{B}_{0} \cdot \mathbf{E}_{1} \\
& -\frac{j \omega_{a}}{2} g_{a \gamma \gamma} \epsilon_{0} \tilde{a} c \vec{B}_{0} \cdot \mathbf{E}_{1}^{*}-\frac{1}{2} \mathbf{E}_{1}^{*} \cdot \mathbf{J}_{e_{1}}+\frac{1}{2} g_{a \gamma \gamma} \tilde{a}^{*} c \vec{B}_{0} \cdot \mathbf{J}_{e_{1}} \\
& -\frac{1}{2} g_{a \gamma \gamma} \tilde{a}^{*} c \mathbf{B}_{1} \cdot \vec{J}_{e_{0}} .
\end{aligned}
$$

Now by substituting (A5) and (A6) into (A1) we obtain

$$
\begin{aligned}
\nabla \cdot j \operatorname{Im}\left(\mathbf{S}_{D B}\right)= & \frac{j \omega_{1}}{2}\left(\frac{1}{\mu_{0}} \mathbf{B}_{1}^{*} \cdot \mathbf{B}_{1}-\epsilon_{0} \mathbf{E}_{1} \cdot \mathbf{E}_{1}^{*}\right) \\
& +\frac{j\left(\omega_{1}+\omega_{a}\right) \epsilon_{0} g_{a \gamma \gamma}}{4} c \vec{B}_{0} \cdot\left(\tilde{a} \mathbf{E}_{1}^{*}+\tilde{a}^{*} \mathbf{E}_{1}\right) \\
& +\frac{1}{4} g_{a \gamma \gamma} c \vec{B}_{0} \cdot\left(\tilde{a} \mathbf{J}_{e_{1}}^{*}-\tilde{a}^{*} \mathbf{J}_{e_{1}}\right) \\
& +\frac{1}{4} g_{a \gamma \gamma} \vec{J}_{e_{0}} \cdot\left(\tilde{a}^{*} c \mathbf{B}_{1}-\tilde{a} c \mathbf{B}_{1}^{*}\right) \\
& -\frac{1}{4}\left(\mathbf{E}_{1} \cdot \mathbf{J}_{e_{1}}^{*}-\mathbf{E}_{1}^{*} \cdot \mathbf{J}_{e_{1}}\right) .
\end{aligned}
$$$$
\nabla \cdot \operatorname{Re}\left(\mathbf{S}_{D B}\right)=\frac{j\left(\omega_{1}-\omega_{a}\right)}{4} \epsilon_{0} g_{a \gamma \gamma} c \vec{B}_{0} \cdot\left(\tilde{a} \mathbf{E}_{1}^{*}-\tilde{a}^{*} \mathbf{E}_{1}\right)
$$$$
+\frac{1}{4} g_{a \gamma \gamma} c \vec{B}_{0} \cdot\left(\tilde{a} \mathbf{J}_{e_{1}}^{*}+\tilde{a}^{*} \mathbf{J}_{e_{1}}\right)
$$$$
-\frac{1}{4} g_{a \gamma \gamma} \vec{J}_{e_{0}} \cdot\left(\tilde{a}^{*} c \mathbf{B}_{1}+\tilde{a} c \mathbf{B}_{1}^{*}\right)
$$$$
-\frac{1}{4}\left(\mathbf{E}_{1} \cdot \mathbf{J}_{e_{1}}^{*}+\mathbf{E}_{1}^{*} \cdot \mathbf{J}_{e_{1}}\right)
$$

and

Then applying the divergence theorem, we arrive at 


$$
\begin{aligned}
\oint & \operatorname{Re}\left(\mathbf{S}_{D B}\right) \cdot \hat{n} d s \\
= & \int\left(\frac{j\left(\omega_{1}-\omega_{a}\right)}{4} \epsilon_{0} g_{a \gamma \gamma} c \vec{B}_{0} \cdot\left(\tilde{a} \mathbf{E}_{1}^{*}-\tilde{a}^{*} \mathbf{E}_{1}\right)\right. \\
& +\frac{1}{4} g_{a \gamma \gamma} c \vec{B}_{0} \cdot\left(\tilde{a} \mathbf{J}_{e_{1}}^{*}+\tilde{a}^{*} \mathbf{J}_{e_{1}}\right) \\
& -\frac{1}{4} g_{a \gamma \gamma} \vec{J}_{e_{0}} \cdot\left(\tilde{a}^{*} c \mathbf{B}_{1}+\tilde{a} c \mathbf{B}_{1}^{*}\right) \\
& \left.-\frac{1}{4}\left(\mathbf{E}_{1} \cdot \mathbf{J}_{e_{1}}^{*}+\mathbf{E}_{1}^{*} \cdot \mathbf{J}_{e_{1}}\right)\right) d V
\end{aligned}
$$

the same as Eq. (27) in the main text, and

$$
\begin{aligned}
& \oint j \operatorname{Im}\left(\mathbf{S}_{D B}\right) \cdot \hat{n} d s \\
& =\int\left(\frac{j \omega_{1}}{2}\left(\frac{1}{\mu_{0}} \mathbf{B}_{1}^{*} \cdot \mathbf{B}_{1}-\epsilon_{0} \mathbf{E}_{1} \cdot \mathbf{E}_{1}^{*}\right)\right. \\
& \quad+\frac{j\left(\omega_{1}+\omega_{a}\right) \epsilon_{0} g_{a \gamma \gamma}}{4} c \vec{B}_{0} \cdot\left(\tilde{a} \mathbf{E}_{1}^{*}+\tilde{a}^{*} \mathbf{E}_{1}\right) \\
& \quad+\frac{1}{4} g_{a \gamma \gamma} c \vec{B}_{0} \cdot\left(\tilde{a} \mathbf{J}_{e_{1}}^{*}-\tilde{a}^{*} \mathbf{J}_{e_{1}}\right)+\frac{1}{4} g_{a \gamma \gamma} \vec{J}_{e_{0}} \cdot\left(\tilde{a}^{*} c \mathbf{B}_{1}-\tilde{a} c \mathbf{B}_{1}^{*}\right) \\
& \left.\quad-\frac{1}{4}\left(\mathbf{E}_{1} \cdot \mathbf{J}_{e_{1}}^{*}-\mathbf{E}_{1}^{*} \cdot \mathbf{J}_{e_{1}}\right)\right) d V,
\end{aligned}
$$

the same as Eq. (28) in the main text.

\section{Axion modified Abraham Poynting theorem}

To derive Eqs. (31) and (32), we begin with writing the divergence of the real and imaginary parts of $\mathbf{S}_{E H}$ as

$$
\begin{aligned}
\nabla \cdot \operatorname{Re}\left(\mathbf{S}_{E H}\right) & =\frac{1}{2} \nabla \cdot\left(\mathbf{S}_{E H}+\mathbf{S}_{E H}^{*}\right), \\
\nabla \cdot \operatorname{Im}\left(\mathbf{S}_{E H}\right) & =\frac{1}{2} \nabla \cdot\left(\mathbf{S}_{E H}-\mathbf{S}_{E H}^{*}\right) .
\end{aligned}
$$

The next step is to calculate $\nabla \cdot \mathbf{S}_{E H}$ and $\nabla \cdot \mathbf{S}_{E H}^{*}$,

$$
\begin{aligned}
\nabla \cdot \mathbf{S}_{E H} & =\frac{1}{2} \nabla \cdot\left(\mathbf{E}_{1} \times \frac{1}{\mu_{0}} \mathbf{B}_{1}^{*}\right) \\
& =\frac{1}{2}\left(\frac{1}{\mu_{0}} \mathbf{B}_{1}^{*} \cdot \nabla \times \mathbf{E}_{1}-\mathbf{E}_{1} \cdot \frac{1}{\mu_{0}} \nabla \times \mathbf{B}_{1}^{*}\right)
\end{aligned}
$$

and

$$
\begin{aligned}
\nabla \cdot \mathbf{S}_{E H}^{*} & =\frac{1}{2} \nabla \cdot\left(\mathbf{E}_{1}^{*} \times \frac{1}{\mu_{0}} \mathbf{B}_{1}\right) \\
& =\frac{1}{2}\left(\frac{1}{\mu_{0}} \mathbf{B}_{1} \cdot \nabla \times \mathbf{E}_{1}^{*}-\mathbf{E}_{1}^{*} \cdot \frac{1}{\mu_{0}} \nabla \times \mathbf{B}_{1}\right) .
\end{aligned}
$$

Considering the Abraham Poynting vector, under a background DC $B$-field of $\vec{B}_{0}(\vec{r})$, created by an impressed electrical DC current in the magnet coil, $\vec{J}_{e_{0}}$, in harmonic form, Ampere's law is modified but Faraday's law is not, and may be written as

$$
\begin{aligned}
\frac{1}{\mu_{0}} \nabla \times \mathbf{B}_{1} & =\mathbf{J}_{e_{1}}-j \omega_{1} \epsilon_{0} \mathbf{E}_{1}+j \omega_{a} g_{a \gamma \gamma} \epsilon_{0} c \vec{B}_{0} \tilde{a}, \\
\frac{1}{\mu_{0}} \nabla \times \mathbf{B}_{1}^{*} & =\mathbf{J}_{e_{1}}^{*}+j \omega_{1} \epsilon_{0} \mathbf{E}_{1}^{*}-j \omega_{a} g_{a \gamma \gamma} \epsilon_{0} c \vec{B}_{0} \tilde{a}^{*}, \\
\nabla \times \mathbf{E}_{1} & =j \omega_{1} \mathbf{B}_{1}, \\
\nabla \times \mathbf{E}_{1}^{*} & =-j \omega_{1} \mathbf{B}_{1}^{*} .
\end{aligned}
$$

Substituting Eq. (A14) into Eqs. (A12) and (A13) leads to

$$
\begin{aligned}
\nabla \cdot \mathbf{S}_{E H}= & \frac{j \omega_{1}}{2}\left(\frac{1}{\mu_{0}} \mathbf{B}_{1}^{*} \cdot \mathbf{B}_{1}-\epsilon_{0} \mathbf{E}_{1} \cdot \mathbf{E}_{1}^{*}\right) \\
& +\frac{j \omega_{a}}{2} g_{a \gamma \gamma} \epsilon_{0} \tilde{a}^{*} c \vec{B}_{0} \cdot \mathbf{E}_{1}-\frac{1}{2} \mathbf{E}_{1} \cdot \mathbf{J}_{e_{1}}^{*}
\end{aligned}
$$

and

$$
\begin{aligned}
\nabla \cdot \mathbf{S}_{E H}^{*}= & \frac{j \omega_{1}}{2}\left(\epsilon_{0} \mathbf{E}_{1} \cdot \mathbf{E}_{1}^{*}-\frac{1}{\mu_{0}} \mathbf{B}_{1}^{*} \cdot \mathbf{B}_{1}\right) \\
& -\frac{j \omega_{a}}{2} g_{a \gamma \gamma} \epsilon_{0} \tilde{a} c \vec{B}_{0} \cdot \mathbf{E}_{1}^{*}-\frac{1}{2} \mathbf{E}_{1}^{*} \cdot \mathbf{J}_{e_{1}} .
\end{aligned}
$$

Now by substituting (A15) and (A16) into (A11) we obtain

$$
\begin{aligned}
\nabla \cdot \operatorname{Re}\left(\mathbf{S}_{E H}\right)= & \frac{j \omega_{a}}{4} \epsilon_{0} g_{a \gamma \gamma} c \vec{B}_{0} \cdot\left(\tilde{a}^{*} \mathbf{E}_{1}-\tilde{a} \mathbf{E}_{1}^{*}\right) \\
& -\frac{1}{4}\left(\mathbf{E}_{1} \cdot \mathbf{J}_{e_{1}}^{*}+\mathbf{E}_{1}^{*} \cdot \mathbf{J}_{e_{1}}\right)
\end{aligned}
$$

and

$$
\begin{aligned}
\nabla \cdot j \operatorname{Im}\left(\mathbf{S}_{E H}\right)= & \frac{j \omega_{1}}{2}\left(\frac{1}{\mu_{0}} \mathbf{B}_{1}^{*} \cdot \mathbf{B}_{1}-\epsilon_{0} \mathbf{E}_{1} \cdot \mathbf{E}_{1}^{*}\right) \\
& +\frac{j \omega_{a}}{4} \epsilon_{0} g_{a \gamma \gamma} c \vec{B}_{0} \cdot\left(\tilde{a}^{*} \mathbf{E}_{1}+\tilde{a} \mathbf{E}_{1}^{*}\right) \\
& -\frac{1}{4}\left(\mathbf{E}_{1} \cdot \mathbf{J}_{e_{1}}^{*}-\mathbf{E}_{1}^{*} \cdot \mathbf{J}_{e_{1}}\right) .
\end{aligned}
$$

Then applying the divergence theorem, we arrive at

$$
\begin{aligned}
\oint \operatorname{Re}\left(\mathbf{S}_{E H}\right) \cdot \hat{n} d s= & \int\left(\frac{j \omega_{a}}{4} \epsilon_{0} g_{a \gamma \gamma} c \vec{B}_{0} \cdot\left(\tilde{a}^{*} \mathbf{E}_{1}-\tilde{a} \mathbf{E}_{1}^{*}\right)\right) \\
& \left.-\frac{1}{4}\left(\mathbf{E}_{1} \cdot \mathbf{J}_{e_{1}}^{*}+\mathbf{E}_{1}^{*} \cdot \mathbf{J}_{e_{1}}\right)\right) d V, \quad(\mathrm{~A} 19)
\end{aligned}
$$

the same as Eq. (31) in the main text, and 


$$
\begin{aligned}
\oint j \operatorname{Im}\left(\mathbf{S}_{E H}\right) \cdot \hat{n} d s= & \int\left(\frac{j \omega_{1}}{2}\left(\frac{1}{\mu_{0}} \mathbf{B}_{1}^{*} \cdot \mathbf{B}_{1}-\epsilon_{0} \mathbf{E}_{1} \cdot \mathbf{E}_{1}^{*}\right)\right. \\
& +\frac{j \omega_{a}}{4} \epsilon_{0} g_{a \gamma \gamma} c \vec{B}_{0} \cdot\left(\tilde{a}^{*} \mathbf{E}_{1}+\tilde{a} \mathbf{E}_{1}^{*}\right) \\
& \left.\left.-\frac{1}{4}\left(\mathbf{E}_{1} \cdot \mathbf{J}_{e_{1}}^{*}-\mathbf{E}_{1}^{*} \cdot \mathbf{J}_{e_{1}}\right)\right)\right) d V, \quad(\mathrm{~A}
\end{aligned}
$$

the same as Eq. (32) in the main text.

\section{APPENDIX B: CONSIDERATION OF THE REACTIVE POWER FLOW IN A RESONANT HALOSCOPE}

In this Appendix we consider the impact of the reactive part of the Poynting vector on a resonant cavity axion haloscope. In general, reactive coupling of power into a resonant cavity may be calculated by implementing Foster's reactance theorem $[123,124,134]$. Foster showed that a lossless circuit network made of resonances and antiresonances could be represented as a combination of inductors and capacitors [124], and following this Beringer and Dicke applied the theorem to high- $Q$ microwave cavities $[123,134]$, allowing the calculation of the effect of reactive coupling to a cavity, based on the complex Poynting theorem [122]. In general, it has been shown that the reactive coupling network into a high- $Q$ resonance may be represented by either an impedance, $\mathcal{X}_{1}$, in series with a parallel LC circuit (of elements $L_{p 1}, C_{p i}$, and $R_{p 1}$ ), or an admittance, $\mathcal{B}_{1}$, in parallel with a series LC circuit (of elements $L_{s 1}, C_{s 1}$, and $R_{s 1}$ ). Applying this technique to axion-photon conversion in a resonant haloscope leads to the following equivalent circuit shown in Fig. 3.

Applying Foster's reactance theorem to the cavity resonator allows $\mathcal{X}_{1}$ and $\mathcal{B}_{1}$ to be calculated from the following equations [134]:

$j \mathcal{X}_{1}=j \omega_{1} \frac{4\left(U_{B 1}-U_{E 1}\right)}{\tilde{I} \tilde{I}^{*}}$ and $j \mathcal{B}_{1}=j \omega_{1} \frac{4\left(U_{E 1}-U_{B 1}\right)}{\tilde{\mathcal{V}} \tilde{\mathcal{V}}^{*}}$

where

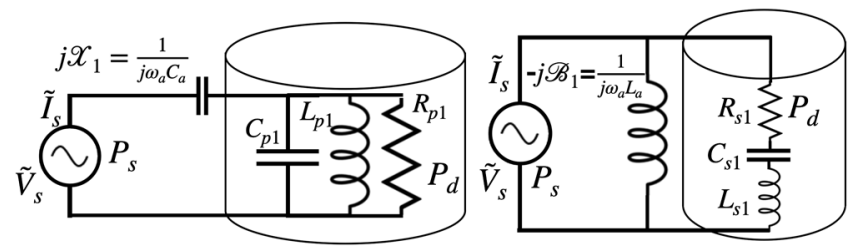

FIG. 3. Left, equivalent parallel $L C R$ circuit representation of an axion coupling to a resonant cavity haloscope. Right, the equivalent series $L C R$ circuit representation.
$U_{B 1}=\frac{1}{4 \mu_{0}} \int \mathbf{B}_{1}^{*} \cdot \mathbf{B}_{1} d V \quad$ and $\quad U_{E 1}=\frac{\epsilon_{0}}{4} \int \mathbf{E}_{1} \cdot \mathbf{E}_{1}^{*} d V$.

Following this procedure, the axion-photon coupling input impedances for both the axion modified Abraham Poynting Vector and the axion modified Minkowski Poynting Vector may be calculated, and this is undertaken in the following sections.

\section{Abraham Poynting theorem}

To calculate the parameters for the parallel $L C R$ circuit shown in Fig. 3, Eqs. (B1) and (32) are combined, and given that the real part of $\mathbf{E}_{1}$ is out of phase with any conduction currents in the volume, and zero at the cavity boundary, then the series impedance becomes

$$
j \mathcal{X}_{1}=-j \frac{\omega_{a}}{2} \frac{\int \epsilon_{0} g_{a \gamma \gamma} c \vec{B}_{0} \cdot\left(\tilde{a}^{*}+\tilde{a}\right) \operatorname{Re}\left(\mathbf{E}_{1}\right) d V}{\tilde{I}^{*}} .
$$

Then given that the energy in a LC resonator is given by $U_{1}=\frac{1}{2} \tilde{I} \tilde{I}^{*} L_{p 1}$, where $\omega_{1}^{2}=\frac{1}{L_{p 1} C_{p 1}}$, and using the result from Eq. (37), Eq. (B3) becomes

$j \mathcal{X}_{1}=\frac{\omega_{a}}{j \omega_{1}^{2} C_{p 1}} \frac{\frac{a_{0} \epsilon_{0} g_{a \gamma \gamma} c}{2} \int \vec{B}_{0} \cdot \operatorname{Re}\left(\mathbf{E}_{1}\right) d V}{U_{1}}=\frac{\omega_{a}}{j \omega_{1}^{2} C_{p 1}}$,

which is equivalent to a capacitance of $C_{a}=C_{p 1} \frac{\omega_{1}^{2}}{\omega_{a}^{2}}$. Thus, the input impedance, $Z_{p}\left(\omega_{a}\right)$, of the parallel circuit representation can be written in normalized form as

$$
\frac{Z_{p}\left(\omega_{a}\right)}{R_{p 1}}=\frac{\omega_{a}}{j \omega_{1} Q_{1}}+\frac{1}{1+j Q_{1}\left(\frac{\omega_{a}}{\omega_{1}}-\frac{\omega_{1}}{\omega_{a}}\right)},
$$

where $Q_{1}=\omega_{1} R_{p 1} C_{p 1}$. Defining the detuning as $\delta \omega=\omega_{a}-\omega_{1}$, where $\delta \omega \ll \omega_{1}$ and $\delta_{a}=\frac{\delta \omega}{\omega_{1}}$, then

$$
\frac{Z_{p}\left(\delta_{a}\right)}{R_{p 1}} \approx \frac{1-j 2 Q_{1}\left(\delta_{a}+\frac{1}{2 Q_{1}^{2}}\right)}{1+4 Q_{1}^{2} \delta_{a}^{2}} .
$$

Setting the imaginary part to zero allows the calculation of the frequency shift of the resonant mode due to the axion coupling, which gives $\frac{\delta \omega_{1}}{\omega_{1}} \sim-\frac{1}{2 Q_{1}^{2}}$ a very small frequency shift, which to first order does not affect the sensitivity of the axion haloscope and is the same order as a frequency shift due to dissipation. Ignoring this term gives the usual complex response of a resonant LCR circuit.

To calculate the parameters for the series $L C R$ circuit shown in Fig. 3, we can use a similar procedure given $U_{1}=\frac{1}{2} \tilde{\mathcal{V}} \tilde{\mathcal{V}}^{*} C_{s 1}$, where $\omega_{1}=\frac{1}{L_{s 1} C_{s 1}}$, along with Eqs. (B1) and (32) to show that the parallel input admittance is given by 


$$
-j \mathcal{B}_{1}=\frac{\omega_{a}}{j \omega_{1}^{2} L_{s 1}} \frac{\frac{a_{0} \varepsilon_{0} g_{a \gamma \gamma} c}{2} \int \vec{B}_{0} \cdot \operatorname{Re}\left(\mathbf{E}_{1}\right) d V}{U_{1}}=\frac{\omega_{a}}{j \omega_{1}^{2} L_{s 1}},
$$

which is equivalent to an inductance of $L_{a}=L_{s 1} \frac{\omega_{1}^{2}}{\omega_{a}^{2}}$. Thus, the input admittance, $Y_{s}\left(\omega_{a}\right)$, of the series circuit representation may be written in normalized form as

$$
R_{s 1} Y_{s}\left(\omega_{a}\right)=\frac{\omega_{a}}{j \omega_{1} Q_{1}}+\frac{1}{1+j Q_{1}\left(\frac{\omega_{a}}{\omega_{1}}-\frac{\omega_{1}}{\omega_{a}}\right)},
$$

where $Q_{1}=\frac{\omega_{1} L_{s 1}}{R_{s 1}}$, so

$$
R_{s 1} Y_{s}\left(\omega_{a}\right) \approx \frac{1-j 2 Q_{1}\left(\delta_{a}+\frac{1}{2 Q_{1}^{2}}\right)}{1+4 Q_{1}^{2} \delta_{a}^{2}},
$$

which completes the dual representation of the resonant axion haloscope as either a parallel $L C R$ in series with a capacitive coupling element or a series $L C R$ circuit in parallel with an inductive coupling element.

\section{Minkowski Poynting theorem}

The reactive part of the Minkowski Poynting vector as written in Eq. (28) has extra terms compared to the Abraham Poynting vector, and by following a similar process, the equivalent equation for the series impedance can be calculated to be

$$
\begin{aligned}
& j \mathcal{X}_{1 M}=\frac{\omega_{a}+\omega_{1}}{j \omega_{1}^{2} C_{p 1}} \frac{\frac{\epsilon_{0} g_{a \gamma} a_{0} c}{2} \int \vec{B}_{0} \cdot \operatorname{Re}\left(\mathbf{E}_{1}\right) d V}{U_{1}} \\
& +\frac{1}{4 \omega_{1}^{2} C_{p 1}} \frac{g_{a \gamma \gamma} c \int \vec{B}_{0} \cdot\left(\tilde{a}^{*} \mathbf{J}_{e_{1}}-\tilde{a} \mathbf{J}_{e_{1}}^{*}\right) d V}{U_{1}} .
\end{aligned}
$$

The second term is nonzero due to lossless inductive currents at the cavity surface, $\kappa_{e_{1}}$, which are in phase with the magnetic field, $\mathbf{B}_{1}$, and related by $\kappa_{e 1}=\frac{1}{\mu_{0}} \hat{n} \times \mathbf{B}_{1}$, where $\hat{n}$ is the normal to the cavity surface, and because the surface current and magnetic field are in imaginary phase, then $\kappa_{e_{1}}^{*}=-\kappa_{e_{1}}$. Note $\mathbf{J}_{e_{1}}=0$, over the volume, unless there is loss in the volume, which contributes to the real part of the Poynting vector, not the reactive part. Next, by implementing the identity, $\oint d \vec{s} \times \mathbf{B}_{1}=\int \nabla \times \mathbf{B}_{1} d V$, and from Eq. (18), to first order we may substitute the following, $\nabla \times \mathbf{B}_{1} \rightarrow-j \omega_{1} \epsilon_{0} \mathbf{E}_{1}$ (ignoring terms second order in $g_{a \gamma \gamma}$ ). Then it is straightforward to show (given $\vec{d} s=d s \hat{n})$

$$
\begin{aligned}
& \vec{B}_{0} \cdot \int\left(\tilde{a}^{*} \mathbf{J}_{e_{1}}-\tilde{a} \mathbf{J}_{e_{1}}^{*}\right) d V=2 a_{0} \vec{B}_{0} \cdot \oint \kappa_{1} d s \\
& =2 a_{0} \vec{B}_{0} \cdot \oint \vec{d} s \times \mathbf{B}_{1}=-2 j a_{0} \omega_{1} \int \vec{B}_{0} \cdot \operatorname{Re}\left(\mathbf{E}_{1}\right) d V,
\end{aligned}
$$

for the resonant cavity haloscope. Therefore substituting Eq. (B11) into (B10), the series impedance becomes

$$
j \mathcal{X}_{1 M}=\frac{\omega_{a}+2 \omega_{1}}{j \omega_{1}^{2} C_{p 1}} .
$$

So the effective input capacitance represented in Fig. 3 becomes $C_{a}=C_{p 1} \frac{\omega_{1}}{\omega_{a}\left(2+\frac{\omega_{a}}{\omega_{1}}\right)}$, which is about a factor of 3 smaller than $C_{a}$ for the Abraham equivalent circuit when $\omega_{a} \sim \omega_{1}$. The normalized input impedance to first order in $\delta_{a}$ can thus be written as

$$
\frac{Z_{p}\left(\delta_{a}\right)}{R_{p 1}} \approx \frac{1-j 2 Q_{1}\left(\delta_{a}+\frac{3}{2 Q_{1}^{2}}\right)}{1+4 Q_{1}^{2} \delta_{a}^{2}} .
$$

Setting the imaginary part to zero allows the calculation of the frequency shift of the resonant mode due to the axion coupling, which gives $\frac{\delta \omega_{1}}{\omega_{1}} \sim-\frac{3}{2 Q_{1}^{2}}$ a very small frequency shift but a factor of 3 greater than what the Abraham Poynting vector predicts. A precision frequency measurement of the axion interacting with a microwave cavity haloscope would be needed to determine this frequency shift.

A similar calculation can be undertaken for the effective parallel inductance for the series $L C R$ circuit representation, the end result is an inductance of $L_{a}=L_{s 1} \frac{\omega_{1}}{\omega_{a}\left(2+\frac{\omega_{a}}{\omega_{1}}\right)}$ leading to similar conclusions and a normalized input admittance of

$$
R_{s 1} Y_{s}\left(\omega_{a}\right) \approx \frac{1-j 2 Q_{1}\left(\delta_{a}+\frac{3}{2 Q_{1}^{2}}\right)}{1+4 Q_{1}^{2} \delta_{a}^{2}},
$$

which completes our analysis. 
[1] R. D. Peccei and H. R. Quinn, Cp Conservation in the Presence of Pseudoparticles, Phys. Rev. Lett. 38, 1440 (1977).

[2] F. Wilczek, Problem of Strong $p$ and $t$ Invariance in the Presence of Instantons, Phys. Rev. Lett. 40, 279 (1978).

[3] S. Weinberg, A New Light Boson?, Phys. Rev. Lett. 40, 223 (1978).

[4] J. Jaeckel and A. Ringwald, The low-energy frontier of particle physics, Annu. Rev. Nucl. Part. Sci. 60, 405 (2010).

[5] J. E. Kim, Weak-Interaction Singlet and Strong CP Invariance, Phys. Rev. Lett. 43, 103 (1979).

[6] J.E. Kim and G. Carosi, Axions and the strong $c p$ problem, Rev. Mod. Phys. 82, 557 (2010).

[7] A. R. Zhitnitsky, On possible suppression of the axion hadron interactions (in russian), Sov. J. Nucl. Phys. 31, 260 (1980).

[8] M. Dine, W. Fischler, and M. Srednicki, A simple solution to the strong $\{\mathrm{CP}\}$ problem with a harmless axion, Phys. Lett. 104B, 199 (1981).

[9] M. A. Shifman, A. I. Vainshtein, and V. I. Zakharov, Can confinement ensure natural $\{\mathrm{CP}\}$ invariance of strong interactions?, Nucl. Phys. B166, 493 (1980).

[10] M. Dine and W. Fischler, The not-so-harmless axion, Phys. Lett. 120B, 137 (1983).

[11] J. Preskill, M. B. Wise, and F. Wilczek, Cosmology of the invisible axion, Phys. Lett. 120B, 127 (1983).

[12] L. F. Abbott and P. Sikivie, A cosmological bound on the invisible axion, Phys. Lett. 120B, 133 (1983).

[13] J. Ipser and P. Sikivie, Can Galactic Halos Be Made of Axions?, Phys. Rev. Lett. 50, 925 (1983).

[14] P. Sikivie, Experimental Tests of the "Invisible" Axion, Phys. Rev. Lett. 51, 1415 (1983).

[15] P. Sikivie, Experimental Tests of the "Invisible" Axion, Phys. Rev. Lett. 52, 695 (1984).

[16] C. Hagmann, P. Sikivie, N. Sullivan, D. B. Tanner, and S.I. Cho, Cavity design for a cosmic axion detector, Rev. Sci. Instrum. 61, 1076 (1990).

[17] C. Hagmann, P. Sikivie, N. S. Sullivan, and D. B. Tanner, Results from a search for cosmic axions, Phys. Rev. D 42, 1297 (1990).

[18] R. Bradley, J. Clarke, D. Kinion, L. J. Rosenberg, K. van Bibber, S. Matsuki, M. Muck, and P. Sikivie, Microwave cavity searches for dark-matter axions, Rev. Mod. Phys. 75, 777 (2003).

[19] S. J. Asztalos, G. Carosi, C. Hagmann, D. Kinion, K. van Bibber, M. Hotz, L. J. Rosenberg, G. Rybka, J. Hoskins, J. Hwang, P. Sikivie, D. B. Tanner, R. Bradley, and J. Clarke, Squid-Based Microwave Cavity Search for Dark-Matter Axions, Phys. Rev. Lett. 104, 041301 (2010).

[20] J. Hoskins, J. Hwang, C. Martin, P. Sikivie, N. S. Sullivan, D. B. Tanner, M. Hotz, L. J Rosenberg, G. Rybka, A. Wagner, S. J. Asztalos, G. Carosi, C. Hagmann, D. Kinion, K. van Bibber, R. Bradley, and J. Clarke, Search for nonvirialized axionic dark matter, Phys. Rev. D 84, 121302 (2011).

[21] T. Braine et al., Extended Search for the Invisible Axion with the Axion Dark Matter Experiment, Phys. Rev. Lett. 124, 101303 (2020).
[22] C. Bartram et al. Axion dark matter experiment: Run 1b analysis details, Phys. Rev. D 103, 032002 (2021).

[23] P. Svrcek and E. Witten, Axions in string theory, J. High Energy Phys. 06 (2006) 051.

[24] A. Arvanitaki, S. Dimopoulos, S. Dubovsky, N. Kaloper, and J. March-Russell, String axiverse, Phys. Rev. D 81, 123530 (2010).

[25] T. Higaki, K. Nakayama, and F. Takahashi, Cosmological constraints on axionic dark radiation from axion-photon conversion in the early universe, J. Cosmol. Astropart. Phys. 09 (2013) 030.

[26] D. Baumann, D. Green, and B. Wallisch, New Target for Cosmic Axion Searches, Phys. Rev. Lett. 117, 171301 (2016).

[27] R. T. Co, L. J. Hall, and K. Harigaya, Axion Kinetic Misalignment Mechanism, Phys. Rev. Lett. 124, 251802 (2020).

[28] R. T. Co and K. Harigaya, Axiogenesis, Phys. Rev. Lett. 124, 111602 (2020).

[29] R. T. Co, L. J. Hall, and K. Harigaya, Predictions for axion couplings from ALP cogenesis, J. High Energy Phys. 1 (2021) 172.

[30] V. K. Oikonomou, Unifying inflation with early and late dark energy epochs in axion $f(r)$ gravity, Phys. Rev. D 103, 044036 (2021).

[31] P. Sikivie, Invisible axion search methods, Rev. Mod. Phys. 93, 015004 (2021).

[32] A. V. Sokolov and A. Ringwald, Photophilic hadronic axion from heavy magnetic monopoles, J. High Energy Phys. 6 (2021) 123.

[33] L. Di Luzio, M. Giannotti, E. Nardi, and L. Visinelli, The landscape of QCD axion models, Phys. Rep. 870, 1 (2020).

[34] J. A. Dror, H. Murayama, and N. L. Rodd, Cosmic axion background, Phys. Rev. D 103, 115004 (2021).

[35] A. Payez, C. Evoli, T. Fischer, M. Giannotti, A. Mirizzi, and A. Ringwald, Revisiting the SN1987a gamma-ray limit on ultralight axion-like particles, J. Cosmol. Astropart. Phys. 02 (2015) 006.

[36] B. T. McAllister, S. R. Parker, and M. E. Tobar, Axion Dark Matter Coupling to Resonant Photons via Magnetic Field, Phys. Rev. Lett. 116, 161804 (2016); Erratum, Phys. Rev. Lett. 117, 159901 (2016).

[37] R. Gupta, M. Anerella, A. Ghosh, W. Sampson, J. Schmalzle, D. Konikowska, Y. K. Semertzidis, and Y. Shin, High-field solenoid development for axion dark matter search at CAPP/IBS, IEEE Trans. Appl. Supercond. 2016) 4100705 ,26).

[38] B. T. McAllister, S. R. Parker, and M. E. Tobar, 3D lumped LC resonators as low mass axion haloscopes, Phys. Rev. D 94, 042001 (2016).

[39] B. T. Mcallister, S. R. Parker, E. N. Ivanov, and M. E. Tobar, Cross-correlation signal processing for axion and wisp dark matter searches, IEEE Trans. Ultrason. Ferroelectr. Freq. Control 66, 236 (2019).

[40] Y. Kahn, B. R. Safdi, and J. Thaler, Broadband and Resonant Approaches to Axion Dark Matter Detection, Phys. Rev. Lett. 117, 141801 (2016).

[41] C. P. Salemi, J. W. Foster, J. L. Ouellet, A. Gavin, K. M. W. Pappas, S. Cheng, K. A. Richardson, R. Henning, Y. Kahn, R. Nguyen, N. L. Rodd, B. R. Safdi, and L. 
Winslow, Search for Low-Mass Axion Dark Matter with Abracadabra-10 cm, Phys. Rev. Lett. 127, 081801 (2021).

[42] B. T. McAllister, G. Flower, E. N. Ivanov, M. Goryachev, J. Bourhill, and M.E. Tobar, The organ experiment: An axion haloscope above $15 \mathrm{GHz}$, Phys. Dark Universe 18, 67 (2017).

[43] A. Caldwell, G. Dvali, B. Majorovits, A. Millar, G. Raffelt, J. Redondo, O. Reimann, F. Simon, and F. Steffen, Dielectric Haloscopes: A New Way to Detect Axion Dark Matter, Phys. Rev. Lett. 118, 091801 (2017).

[44] A. J. Millar, G. G. Raffelt, J. Redondo, and F. D. Steffen, Dielectric haloscopes to search for axion dark matter: Theoretical foundations, J. Cosmol. Astropart. Phys. 01 (2017) 061.

[45] A. N. Ioannisian, N. Kazarian, A. J. Millar, and G. G. Raffelt, Axion-photon conversion caused by dielectric interfaces: Quantum field calculation, J. Cosmol. Astropart. Phys. 09 (2017) 005.

[46] B. Majorovits, MADMAX: A new road to axion dark matter detection, J. Phys. Conf. Ser. 1342, 012098 (2020).

[47] B. M. Brubaker, L. Zhong, S. K. Lamoreaux, K. W. Lehnert, and K. A. van Bibber, Haystac axion search analysis procedure, Phys. Rev. D 96, 123008 (2017).

[48] J. Jeong, S. Youn, S. Ahn, J. E. Kim, and Y. K. Semertzidis, Concept of multiple-cell cavity for axion dark matter search, Phys. Lett. B 777, 412 (2018).

[49] I. G. Irastorza and J. Redondo, New experimental approaches in the search for axion-like particles, Prog. Part. Nucl. Phys. 102, 89 (2018).

[50] J. L. Ouellet, C. P. Salemi, J. W. Foster, R. Henning, Z. Bogorad, J. M. Conrad, J. A. Formaggio, Y. Kahn, J. Minervini, A. Radovinsky, N. L. Rodd, B. R. Safdi, J. Thaler, D. Winklehner, and L. Winslow, Design and implementation of the abracadabra-10 cm axion dark matter search, Phys. Rev. D 99, 052012 (2019).

[51] K. Nagano, T. Fujita, Y. Michimura, and I. Obata, Axion Dark Matter Search with Interferometric Gravitational Wave Detectors, Phys. Rev. Lett. 123, 111301 (2019).

[52] M. Goryachev, B. T. McAllister, and M. E. Tobar, Axion detection with precision frequency metrology, Phys. Dark Universe 26, 100345 (2019).

[53] J. Choi, H. Themann, M. J. Lee, B. R. Ko, and Y. K. Semertzidis, First axion dark matter search with toroidal geometry, Phys. Rev. D 96, 061102 (2017).

[54] R. Henning et al., First Results from Abracadabra-10 cm: A Search for Sub- $\mu$ ev Axion Dark Matter, Phys. Rev. Lett. 122, 121802 (2019).

[55] H. Liu, B. D. Elwood, M. Evans, and J. Thaler, Searching for axion dark matter with birefringent cavities, Phys. Rev. D 100 (2019).

[56] D. J. E. Marsh, K. C. Fong, E. W. Lentz, and L. Šmejkal, and M. N. Ali, Proposal to Detect Dark Matter Using Axionic Topological Antiferromagnets, Phys. Rev. Lett. 123, 121601 (2019).

[57] J. Schütte-Engel, D. J. E. Marsh, A. J. Millar, A. Sekine, F. Chadha-Day, S. Hoof, M. N. Ali, K. C. Fong, E. Hardy, and L. Šmejkal, Axion quasiparticles for axion dark matter detection, J. Cosmol. Astropart. Phys. 08 (2021) 066.
[58] M. Lawson, A. J. Millar, M. Pancaldi, E. Vitagliano, and F. Wilczek, Tunable Axion Plasma Haloscopes, Phys. Rev. Lett. 123, 141802 (2019).

[59] V. Anastassopoulos et al. (CAST Collaboration), New cast limit on the axion-photon interaction, Nat. Phys. 13, 584 (2017).

[60] L. Zhong et al., Results from phase 1 of the haystac microwave cavity axion experiment, Phys. Rev. D 97, 092001 (2018).

[61] S. Lee, S. Ahn, J. Choi, B. R. Ko, and Y. K. Semertzidis, Axion Dark matter Search Around 6.7 $\mu \mathrm{eV}$, Phys. Rev. Lett. 124, 101802 (2020).

[62] M. E. Tobar, B. T. McAllister, and M. Goryachev, Broadband electrical action sensing techniques with conducting wires for low-mass dark matter axion detection, Phys. Dark Universe 30, 100624 (2020).

[63] G. B. Gelmini, A. J. Millar, V. Takhistov, and E. Vitagliano, Probing dark photons with plasma haloscopes, Phys. Rev. D 102, 043003 (2020).

[64] A. Berlin, R. T. D’Agnolo, S. A. R. Ellis, C. Nantista, J. Neilson, P. Schuster, S. Tantawi, N. Toro, and K. Zhou, Axion dark matter detection by superconducting resonant frequency conversion, J. High Energy Phys. 7 (2020) 1.

[65] R. Lasenby, Parametrics of electromagnetic searches for axion dark matter, Phys. Rev. D 103, 075007 (2021).

[66] A. V. Gramolin, D. Aybas, D. Johnson, J. Adam, and A. O. Sushkov, Search for axion-like dark matter with ferromagnets, Nat. Phys. 17, 79 (2021).

[67] A. Abeln et al. (The IAXO Collaboration), Conceptual design of babyiaxo, the intermediate stage towards the international axion observatory, J. High Energy Phys. 5 (2021) 137.

[68] C. A. Thomson, B. T. McAllister, M. Goryachev, E. N. Ivanov, and M.E. Tobar, Upconversion Loop Oscillator Axion Detection Experiment: A Precision Frequency Interferometric Axion Dark Matter Search with a Cylindrical Microwave Cavity, Phys. Rev. Lett. 126, 081803 (2021).

[69] C. Gatti, P. Gianotti, C. Ligi, M. Raggi, and P. Valente, Dark matter searches at LNF, Universe 7, 236 (2021).

[70] Y. Kishimoto, Y. Suzuki, I. Ogawa, Y. Mori, and M. Yamashita, Development of a cavity with photonic crystal structure for axion searches, Prog. Theor. Exp. Phys. 2021, 063H01 (2021).

[71] J. A. Devlin, M. J. Borchert, S. Erlewein, M. Fleck, J. A. Harrington, B. Latacz, J. Warncke, E. Wursten, M. A. Bohman, A. H. Mooser, C. Smorra, M. Wiesinger, C. Will, K. Blaum, Y. Matsuda, C. Ospelkaus, W. Quint, J. Walz, Y. Yamazaki, and S. Ulmer, Constraints on the Coupling Between Axionlike Dark Matter and Photons Using an Antiproton Superconducting Tuned Detection Circuit in a Cryogenic Penning Trap, Phys. Rev. Lett. 126, 041301 (2021).

[72] O. Kwon et al., First Results from an Axion Haloscope at Capp Around $10.7 \mu \mathrm{eV}$, Phys. Rev. Lett. 126, 191802 (2021).

[73] K. M. Backes et al., A quantum enhanced search for dark matter axions, Nature (London) 590, 238 (2021).

[74] A. Iwazaki, Axion-radiation conversion by super and normal conductors, Nucl. Phys. B963, 115298 (2021). 
[75] S. Chigusa, T. Moroi, and K. Nakayama, Axion/hiddenphoton dark matter conversion into condensed matter axion, J. High Energy Phys. 8 (2021) 74.

[76] X. Liang, E. Peshkov, L. Van Waerbeke, and A. Zhitnitsky, Proposed network to detect axion quark nugget dark matter, Phys. Rev. D 103, 096001 (2021).

[77] D. J. White and P. L. Overfelt, Poynting's theorems and their relationship to antenna power, Q, and bandwidth, Office of Naval Research NAWCWPNS Technical Publication, 8419 (Naval Air Warfare Center Weapons Division China Lake, CA, 1999), https://apps.dtic.mil/sti/ pdfs/ADA367000.pdf.

[78] K. Zhang and D. Li, Electromagnetic Theory for Microwaves and Optoelectronics, 2nd ed. (Springer, New York, 2008).

[79] J. L. Volakis and K. Sertel, Integral Equation Methods for Electromagnetics (Scitech Publishing Inc., Raleigh, NC, USA, 2012).

[80] C. A. Balanis, Advanced Engineering Electromagnetics (John Wiley, New York, 2012).

[81] R. E. Harrington, Introduction to Electromagnetic Engineering, 2nd ed. (Dover Publications, Inc., New York, 2012).

[82] P. Wu, R. Huang, C. Tischer, A. Jonas, and E.-L. Florin, Direct Measurement of the Nonconservative Force Field Generated by Optical Tweezers, Phys. Rev. Lett. 103, 108101 (2009).

[83] M. V. Berry and P. Shukla, Physical curl forces: Dipole dynamics near optical vortices, J. Phys. A 46, 422001 (2013).

[84] M. Bethune-Waddell and K. J. Chau, Simulations of radiation pressure experiments narrow down the energy and momentum of light in matter, Rep. Prog. Phys. 78, 122401 (2015).

[85] S. Sukhov and A. Dogariu, Non-conservative optical forces, Rep. Prog. Phys. 80, 112001 (2017).

[86] I. Liberal, I. Ederra, R. Gonzalo, and R. W. Ziolkowski, Near-field electromagnetic trapping through curl-spin forces, Phys. Rev. A 87, 063807 (2013).

[87] P. Guha, Curl forces and their role in optics and ion trapping, Eur. Phys. J. D 74, 99 (2020).

[88] D. F. Nelson, Momentum, pseudomomentum, and wave momentum: Toward resolving the Minkowski-Abraham controversy, Phys. Rev. A 44, 3985 (1991).

[89] D. J. Griffiths, Resource letter em-1: Electromagnetic momentum, Am. J. Phys. 80, 7 (2012).

[90] M. Mansuripur, Force, torque, linear momentum, and angular momentum in classical electrodynamics, Appl. Phys. A 123, 653 (2017).

[91] V.P. Torchigin, Dozen arguments in favor of the Minkowski form of the momentum of light in matter, Optik (Stuttgart) 218, 164986 (2020).

[92] R. N. C. Pfeifer, T. A. Nieminen, N. R. Heckenberg, and H. Rubinsztein-Dunlop, Colloquium: Momentum of an electromagnetic wave in dielectric media, Rev. Mod. Phys. 79, 1197 (2007).

[93] U. Leonhardt, Momentum in an uncertain light, Nature (London) 444, 823 (2006).

[94] S. M. Barnett, Resolution of the Abraham-Minkowski Dilemma, Phys. Rev. Lett. 104, 070401 (2010).
[95] M. V. Berry and S. Pragya, Hamiltonian curl forces, Proc. R. Soc. A 471, 20150002 (2015).

[96] P. Strange, Quantized hamiltonian curl forces and squeezed light, J. Phys. A 51, 335303 (2018).

[97] M. V. Berry, Classical and quantum complex hamiltonian curl forces, J. Phys. A 53, 415201 (2020).

[98] A. Drezet, Dual-lagrangian description adapted to quantum optics in dispersive and dissipative dielectric media, Phys. Rev. A 94, 053826 (2016).

[99] A. Drezet, Quantizing polaritons in inhomogeneous dissipative systems, Phys. Rev. A 95, 023831 (2017).

[100] A. Drezet, Equivalence between the hamiltonian and langevin noise descriptions of plasmon polaritons in a dispersive and lossy inhomogeneous medium, Phys. Rev. A 96, 033849 (2017).

[101] P. Kinsler, A. Favaro, and M. W McCall, Four Poynting theorems, Eur. J. Phys. 30, 983 (2009).

[102] H. Minkowski, Die grundgleichungen für die elektromagnetischen vorgänge in bewegten körpern, Mathematische Annalen 68, 472 (1910).

[103] J. C. Garrison and R. Y. Chiao, Canonical and kinetic forms of the electromagnetic momentum in an ad hoc quantization scheme for a dispersive dielectric, Phys. Rev. A 70, 053826 (2004).

[104] M. Abraham, Zur elektrodynamik bewegter körper, Rend. Circ. Matem. Palermo 28, 1 (2009).

[105] M. Abraham, Sullelettrodinamica di minkowski, Rend. Circ. Matem. Palermo 30, 33 (1910).

[106] M. E. Tobar, B. T. McAllister, and M. Goryachev, Electrodynamics of Free- and Bound-Charge Electricity Generators Using Impressed Sources, Phys. Rev. Applied 15, 014007 (2021).

[107] R. K. Vasudevan, Y. Cao, N. Laanait, A. Ievlev, L. Li, J.-C. Yang, Y.-H. Chu, L.-Q. Chen, S. V. Kalinin, and P. Maksymovych, Field enhancement of electronic conductance at ferroelectric domain walls, Nat. Commun. 8, 1318 (2017).

[108] D. I. Pikulin, A. Chen, and M. Franz, Chiral Anomaly from Strain-Induced Gauge Fields in Dirac and Weyl Semimetals, Phys. Rev. X 6, 041021 (2016).

[109] J. Yu, J. Zang, and C.-X. Liu, Magnetic resonance induced pseudoelectric field and giant current response in axion insulators, Phys. Rev. B 100, 075303 (2019).

[110] R. Ilan, A. G. Grushin, and D. I. Pikulin, Pseudo-electromagnetic fields in 3D topological semimetals. Nat. Rev. Phys. 2, 29 (2020).

[111] M. E. Tobar, R. Y. Chiao, and M. Goryachev, Active dipoles, electric vector potential and Berry phase, arXiv:2101.00945.

[112] M. E. Tobar, B. T. McAllister, and M. Goryachev, Modified axion electrodynamics as impressed electromagnetic sources through oscillating background polarization and magnetization, Phys. Dark Universe 26, 100339 (2019).

[113] F. Wilczek, Two Applications of Axion Electrodynamics, Phys. Rev. Lett. 58, 1799 (1987).

[114] Y. Kim, D. Kim, J. Jeong, J. Kim, Y. C. Shin, and Y. K. Semertzidis, Effective approximation of electromagnetism for axion haloscope searches, Phys. Dark Universe 26, 100362 (2019). 
[115] L. Visinelli, Axion-electromagnetic waves, Mod. Phys. Lett. A 28, 1350162 (2013).

[116] A. Asker, Axion Electrodynamics and Measurable Effects in Topological Insulators (Kaerstads University, Karlstad, Sweden, 2018).

[117] R. P. Cameron and S. M. Barnett, Electric-magnetic symmetry and noether's theorem, New J. Phys. 14, 123019 (2012).

[118] R. P. Cameron, S. M. Barnett, and A. M. Yao, Optical helicity, optical spin and related quantities in electromagnetic theory, New J. Phys. 14, 053050 (2012).

[119] K. Y. Bliokh, A. Y. Bekshaev, and F. Nori, Dual electromagnetism: Helicity, spin, momentum and angular momentum, New J. Phys. 15, 033026 (2013).

[120] K. Y. Bliokh, A. Y. Bekshaev, and F. Nori, Corrigendum: Dual electromagnetism: Helicity, spin, momentum, and angular momentum (2013new j. phys.15033026), New J. Phys. 18, 089503 (2016).

[121] S. A. Schelkunoff, Some equivalence theorems of electromagnetics and their application to radiation problems, Bell Syst. Tech. J. 15, 92 (1936).

[122] Principles of Microwave Circuits, edited by C. G. Montgomery, R. H. Dicke, and E. M. Purcell (McGraw-Hill, New York, 1987).

[123] R. H. Dicke, General Microwave Circuit Theorems, Principles of Microwave Circuits (McGraw-Hill, New York, 1987), Chap. 5, pp. 130-161.

[124] R. M. Foster, A reactance theorem, Bell Syst. Tech. J. 3, 259 (1924).
[125] J. Ouellet and Z. Bogorad, Solutions to axion electrodynamics in various geometries, Phys. Rev. D 99, 055010 (2019).

[126] M. Beutter, A. Pargner, T. Schwetz, and E. Todarello, Axion-electrodynamics: A quantum field calculation, J. Cosmol. Astropart. Phys. 02 (2019) 026.

[127] C. Cao and A. Zhitnitsky, Axion detection via topological casimir effect, Phys. Rev. D 96, 015013 (2017).

[128] E. Witten, Dyons of charge $e \theta / 2 \pi$, Phys. Lett. 86B, 283 (1979).

[129] B. T. McAllister, M. Goryachev, J. Bourhill, E. N. Ivanov, and M. E. Tobar, Broadband axion dark matter haloscopes via electric field sensing, arXiv:1803.07755.

[130] S. M. Barnett and R. Loudon, The enigma of optical momentum in a medium, Phil. Trans. R. Soc. A: Math. Phys. Eng. Sci. 368, 927 (2010).

[131] X.-Y. Song, Y.-C. He, A. Vishwanath, and C. Wang, Electric polarization as a nonquantized topological response and boundary luttinger theorem. Phys. Rev. Research 3, 023011 (2021).

[132] X.-L. Qi, T. L. Hughes, and S.-C. Zhang, Topological field theory of time-reversal invariant insulators, Phys. Rev. B 78, 195424 (2008).

[133] D. Fallde and D. Baldomir, On the inner topological pressure within the topological insulators, Ann. Phys. (Berlin) 534, 2100313 (2022).

[134] R. Beringer, Resonant Cavities as Microwave Circuit Elements, Principles of Microwave Circuits (McGrawHill, New York, 1987), Chap. 7, pp. 207-239. 